TI 2018-058/IV

Tinbergen Institute Discussion Paper

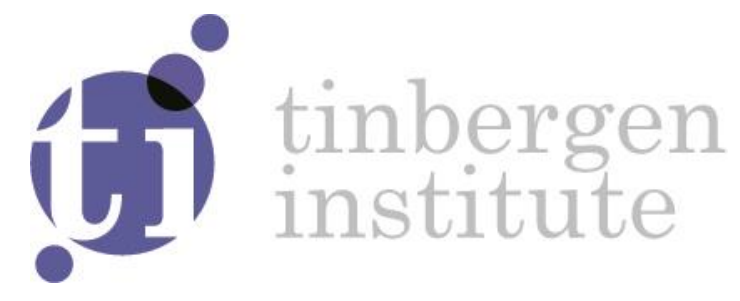

\title{
Measuring Biases in Expectation Formation
}

\author{
Florian Peters ${ }^{1}$ \\ Simas Kucinskas ${ }^{1}$
}

${ }^{1}$ University of Amsterdam, The Netherlands 
Tinbergen Institute is the graduate school and research institute in economics of Erasmus University Rotterdam, the University of Amsterdam and VU University Amsterdam.

Contact: discussionpapers@tinbergen.nl

More TI discussion papers can be downloaded at http://www.tinbergen.nl

Tinbergen Institute has two locations:

Tinbergen Institute Amsterdam

Gustav Mahlerplein 117

1082 MS Amsterdam

The Netherlands

Tel.: +31(0)205984580

Tinbergen Institute Rotterdam

Burg. Oudlaan 50

3062 PA Rotterdam

The Netherlands

Tel.: +31(0)10408 8900 


\title{
Measuring Biases in Expectation Formation*
}

\author{
Simas Kučinskas $^{\dagger} \quad$ Florian S. Peters ${ }^{\ddagger}$
}

May 2018

\begin{abstract}
We develop a general framework for measuring biases in expectation formation. The method is based on the insight that biases can be inferred from the response of forecast errors to past news. Empirically, biases are measured by flexibly estimating the impulse response function of forecast errors. The framework does not require precise knowledge of the true data-generating process, and it nests all major existing models of expectations. Monte Carlo simulations show that the method is able to detect biases in empirically relevant settings. We illustrate the methodology using data on inflation forecasts. Our framework can guide future models of expectations.
\end{abstract}

Keywords: expectation formation; bias; underreaction; overreaction.

JEL Classification: C53, D83, D84, E70, G40.

\footnotetext{
*For comments and suggestions at various stages of the project, we thank Andreas Fuster, Hans-Martin von Gaudecker, Augustin Landier, Ioanid Rosu, Christian Stoltenberg, Paul Tetlock, Uwe Thümmel, and Michael Weber, as well as seminar audiences at the Bank of Lithuania, HEC Paris, University of Amsterdam, University of Bonn, and Vrije Universiteit Amsterdam. Replication files (with Python and STATA code) are available online.

†University of Amsterdam (e-mail: s.kucinskas@uva.nl, website: simaskucinskas.com).

†University of Amsterdam (e-mail: f.s.peters@uva.nl, website: florianpeters.org).
} 


\section{Introduction}

There is ample evidence that subjective expectations deviate from simple forms of rational expectations. ${ }^{1}$ However, there is little agreement on how subjective expectations are actually formed. The lack of consensus has led to a proliferation of models, some of them taking very different views on expectation formation. For instance, much research in macroeconomics has focused on models featuring underreaction to new information. ${ }^{2}$ At the same time, many prominent models in financial economics exhibit overreaction. ${ }^{3}$ Even some of the empirical evidence seems conflicting, with some findings supporting underreaction, and others more consistent with overreaction. ${ }^{4}$

In this paper we propose a general framework for measuring biases in expectation formation. Our proposed method has several attractive features. First, the method does not require precise knowledge of the true data-generating process. Second, the framework is able to capture rich forms of bias such as overreaction to recent news and underreaction to news that occurred far in the past. Finally, our method is straightforward to apply empirically. The method can be used whenever both expectations and realized values are observed, and the time series dimension of the dataset is large enough.

The basic insight is that biases can be inferred from the autocorrelation structure of forecast errors. To be concrete, consider an analyst who is forecasting future inflation. For some reason, inflation in the current quarter is higher than was expected by the analyst. If the analyst reacts to new information optimally, the forecast error in the current quarter should not be predictive of future forecast errors. However, suppose that the analyst does not follow macroeconomic developments very carefully and tends to underreact to news. Since inflation is persistent, a positive forecast error today implies that the forecast error next quarter is likely to again be positive. As a result, underreaction leads to positively autocorrelated forecast errors. In contrast, if the analyst overreacts to

1 For surveys, see Pesaran and Weale (2006, Section 5) and Manski (2018). Coibion, Gorodnichenko, and Kamdar (2018) provide another recent overview, focusing on inflation expectations. We use the adjective "simple" because even if agents are rational and have full information, their forecasts may differ from the true conditional expectations (e.g., if they have asymmetric loss functions, see Patton and Timmermann (2007) and references therein).

2 Examples include sticky information (Mankiw and Reis, 2002), rational inattention (Sims, 2003), imperfect information (e.g., Woodford, 2003), and sparsity-based models of limited attention (Gabaix, 2017b, 2018).

3 Examples include diagnostic expectations (Gennaioli and Shleifer, 2010; Bordalo, Gennaioli, and Shleifer, 2018), extrapolative expectations (e.g., Cutler, Poterba, and Summers, 1990b; DeLong, Shleifer, Summers, and Waldmann, 1990; Barberis, Greenwood, Jin, and Shleifer, 2015), and overconfidence (e.g., Daniel, Hirshleifer, and Subrahmanyam, 1998; Odean, 1998).

4 A striking example is given by De Bondt and Thaler (1990) and Abarbanell and Bernard (1992). These papers provide evidence suggestive of both overreaction (De Bondt and Thaler) and underreaction (Abarbanell and Bernard) for the case of stock market analysts. See also Bouchaud, Krüger, Landier, and Thesmar (2018). 
the higher-than-expected inflation, inflation in the next quarter will on average be lower than anticipated. With overreaction, forecast errors tend to be negatively autocorrelated.

We take this logic to its natural conclusion by representing biases in expectation formation as an impulse response function (IRF) of forecast errors. The IRF of forecast errors yields a set of bias coefficients which provide a natural measure of under- or overreaction to news at various lags. If expectations react to news in an unbiased way, a shock to the forecast errors should die out immediately. If the shock does not die out immediately, expectations are biased. Since we do not impose any structure on the bias coefficients, the method is able to capture rich forms of bias. Empirically, we use local projections (Jordá, 2005) to estimate the IRF of forecast errors without imposing strong parametric assumptions.

The estimated bias coefficients could have multiple economic interpretations. Nonzero bias coefficients only provide evidence of statistical bias. For example, the inflation analyst may underreact to news about inflation because of informational frictions, psychological biases, or career concerns. Additional structure is necessary to distinguish between these competing explanations. Nevertheless, if we choose a particular model of expectations and postulate a process for the variable being forecast, it is straightforward to derive the predicted bias coefficients. Comparing the estimated bias coefficients to their theoretical counterparts provides a natural test of the model. Bias coefficients also provide a natural set of moments to target for calibration exercises or structural estimation.

The existing literature on expectations is voluminous, and we refer to the surveys cited above for comprehensive reviews. We are certainly not the first to study the autocorrelation of forecast errors. A key result in the literature on forecast evaluation is that with optimal one-step-ahead forecasts, the resulting forecast errors are white noise (see, e.g., Diebold and Lopez, 1996). Our contribution is to show that the structure of autocorrelation, not just its existence, is informative about how expectations are formed. In the paper that introduced rational expectations, Muth (1961, pp. 321-322) already considered a model of biased expectation formation that is a special case of our framework. More recently, Coibion and Gorodnichenko (2012) proposed an empirical technique that is related to our method but requires more information about the underlying process. We discuss these and other related papers in detail in Section 2.4 after presenting our framework.

Related issues have also been extensively studied outside economics, most prominently in psychology. The psychology literature has documented a number of biases in the way people form subjective beliefs. Experimental studies of Bayesian updating have found that subjects often do not update enough, a finding known as conservatism 
bias (Edwards, 1968). Studies on belief persistence (also known as belief perseverance) show that people often hold on to incorrect beliefs (see, e.g., Nickerson, 1998, pp. 187188). Conservatism bias and belief persistence are both forms of underreaction to new information. However, other well-known findings in psychology are more consistent with overreaction. For example, Kahneman and Tversky (1973) find that subjects fail to incorporate base rates and the reliability of the available information when making predictions. The famous hot-hand fallacy study of Gilovich, Vallone, and Tversky (1985) suggests that people overreact to noise. ${ }^{5}$

The general framework is developed in Section 2. The basic setting is that of an agent forecasting some variable of interest. We consider expectations that can be represented as a linear combination of past shocks. The key result is that biases in expectation formation are given by the IRF of the forecast errors.

In Section 3 we show that the framework is sufficiently flexible to nest all major existing models of expectations. As a side benefit, the IRF representation of biases provides a new lens to look at existing models. Section 4 discusses a number of extensions to the basic framework, including multiple-step-ahead forecasts, heterogeneity in expectations, measurement error, and multiple shocks and forecasts. We provide a Monte Carlo study in Section 5 to gauge the statistical power of our procedure and investigate its performance when some of the assumptions are violated.

In Section 6 we apply the method to data on inflation forecasts from the Survey of Professional Forecasters. The results show underreaction for up to one year after the arrival of news. We also discuss how the estimated bias coefficients can be used to distinguish between different models of expectations. Section 7 concludes by discussing limitations of the proposed methodology and outlining potential directions for future work.

\footnotetext{
5 This interpretation has recently been challenged by Miller and Sanjurjo (2018). Other classic findings in psychology suggestive of overreaction include illusion of choice (Langer, 1975) and illusory correlation (for a review, see Chapman and Chapman, 1982); Andreassen (1987, p. 490) provides additional references highlighting the tension between under- and overreaction in sequential settings. Griffin and Tversky (1992) argue that the conflicting results can be reconciled if people focus too much on how diagnostic a piece of information is about a given hypothesis but place too little emphasis on the credence of that information. See Nisbett and Ross (1980, especially Chapters 5, 7, and 8) for further discussion.
} 


\section{Methodology}

\subsection{Framework}

We want to measure biases in how an agent forms expectations ${ }^{6}$ about some variable $x_{t}$. For now, we assume that $x_{t}$ is covariance stationary. ${ }^{7}$ To simplify notation, we suppose that any deterministic component in $x_{t}$ has been removed, and the variable is demeaned.

By Wold's Representation Theorem (e.g., Hamilton, 1994, Proposition 4.1),

$$
x_{t}=\sum_{\ell=0}^{+\infty} \alpha_{\ell} \varepsilon_{t-\ell}
$$

for some coefficients satisfying $\alpha_{0}=1$ and $\sum_{\ell=0}^{+\infty} \alpha_{\ell}^{2}<+\infty$, and a white noise series $\varepsilon_{t}$ (i.e., $\mathbb{E}\left[\varepsilon_{t}\right]=0, \mathbb{E}\left[\varepsilon_{t}^{2}\right]=\sigma^{2}$, and $\mathbb{E}\left[\varepsilon_{t} \varepsilon_{s}\right]=0$ for $t \neq s$ ). For the representation above to hold, it is only necessary that $x_{t}$ be covariance stationary (i.e., the first two unconditional moments of $x_{t}$ cannot depend on $t$ ). In particular, the specification does not presume that $x_{t}$ cannot be predicted by other variables.

We observe an agent making one-step ahead forecasts of $x_{t+1} \cdot{ }^{8}$ Denote these forecasts by $\mathbb{F}_{t}\left[x_{t+1}\right]$. We assume that forecasts are generated as

$$
\mathbb{F}_{t}\left[x_{t+1}\right]=b_{0}+\sum_{\ell=0}^{+\infty} a_{\ell+1} \varepsilon_{t-\ell}
$$

Here $b_{0}$ is a time-invariant bias term, while the coefficients $a_{\ell}$ capture how subjective expectations react to past shocks. If $a_{\ell} \neq \alpha_{\ell}$, the subjective reaction to past shocks is different from the reaction of the true process.

The specification in Eq. (2) is flexible and, as shown in Section 3, nests all commonly used models of expectations. The flexibility is essential since there is no standard model of non-rational expectations. In addition, biases may vary across contexts and variables being forecast. For these reasons, we wish to impose as little structure as possible.

Let $\mathbb{E}_{t-1}\left[x_{t}\right]-\mathbb{F}_{t-1}\left[x_{t}\right]$ be the ex-ante forecast error and denote the ex-post forecast error by $e_{t} \equiv x_{t}-\mathbb{F}_{t-1}\left[x_{t}\right]$. (Whenever we write "forecast error" without additional

\footnotetext{
6 We use the terms "expectations" and "forecasts" interchangeably. In applications, observed forecasts need not represent true subjective expectations. In that case, our method will reveal biases of the observed forecasts.

7 The method can be generalized to non-stationary $x_{t}$ as discussed in Section 2.3. As always, even if $x_{t}$ is not stationary, it often can be made stationary by means of simple transformations (e.g., taking first differences or growth rates).

${ }^{8}$ It is straightforward to allow for multiple-step-ahead forecasts, as shown in Section 4.1. As discussed in Section 4.2, when our method is applied to consensus (e.g., average) forecasts, it recovers average biases in expectation formation.
} 
qualifications, we mean the "ex-post forecast error.") We say that expectations are unbiased if the ex-ante forecast error is zero with probability one. This notion of bias is a statistical one. As shown in Section 3, there may be multiple reasons for why expectations may be biased according to this definition, including psychological as well as non-psychological ones. The definition of bias used in the present paper is more stringent than what is meant by bias in some of the existing literature (such as whether forecasts are equal to the realized values on average). Finally, we say that an agent overreacts to shock $\varepsilon_{t-\ell}$ if the perceived response $a_{\ell+1}$ is larger than the true response $\alpha_{\ell+1}$ in absolute value, i.e., $\left|a_{\ell+1}\right|>\left|\alpha_{\ell+1}\right|$. Underreaction is defined in the same way, with the inequality reversed.

With these definitions at hand, we can write the ex-ante forecast error as

$$
\mathbb{E}_{t-1}\left[x_{t}\right]-\mathbb{F}_{t-1}\left[x_{t}\right]=-b_{0}-\sum_{\ell=1}^{+\infty} \operatorname{sgn}\left(\alpha_{\ell}\right) b_{\ell} \varepsilon_{t-\ell}
$$

where $b_{\ell} \equiv \operatorname{sgn}\left(\alpha_{\ell}\right)\left(a_{\ell}-\alpha_{\ell}\right), \ell \geq 1$ stands for the bias coefficients. The sign function, $\operatorname{sgn}\left(\alpha_{\ell}\right)$, is equal to -1 if $\alpha_{\ell}<0$ and 1 otherwise. For the ex-ante forecast error to be zero with probability one, all bias coefficients must equal zero. Hence, the bias coefficients provide a natural measure of bias in expectation formation. The agent overreacts to shocks that arrived $\ell$ periods before the forecast is made if $b_{\ell+1}>0$ and underreacts if $b_{\ell+1}<0$ (if $\alpha_{\ell} \neq 0$ ). For the special case $\alpha_{\ell}=0$, any non-zero bias coefficient indicates overreaction.

Outside experimental settings, we are unlikely to know exactly how $x_{t}$ is generated. As a result, we typically do not observe either the true conditional expectation or the shocks. The main insight of the paper is that even then, the bias coefficients can be inferred from the autocorrelation structure of the ex-post forecast errors. Since $x_{t}=$ $\mathbb{E}_{t-1}\left[x_{t}\right]+\varepsilon_{t}$, Eq. (3) implies that

$$
\underbrace{x_{t}-\mathbb{F}_{t-1}\left[x_{t}\right]}_{e_{t}}=-b_{0}-\sum_{\ell=1}^{+\infty} \operatorname{sgn}\left(\alpha_{\ell}\right) b_{\ell} \varepsilon_{t-\ell}+\varepsilon_{t} .
$$

As a result, estimating the bias coefficients is equivalent to estimating the impulse response function (IRF) of the ex-post forecast errors. To say whether agents underor overreact to new information, it is necessary to have some knowledge of the true process - namely, the sign of $\alpha_{\ell}$. For simply testing whether expectations are unbiased, the sign of $\alpha_{\ell}$ is not needed. As discussed in Section 2.2, the IRF is inferred from the autocorrelation structure of the observed forecast errors.

Figure 1 illustrates the main idea of the method. The dashed blue line shows the IRF 


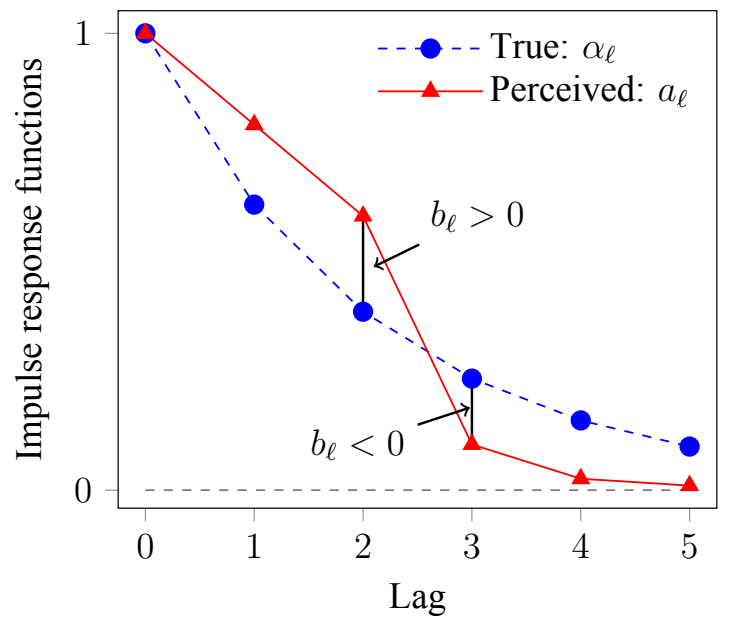

(a) Positive autocorrelation

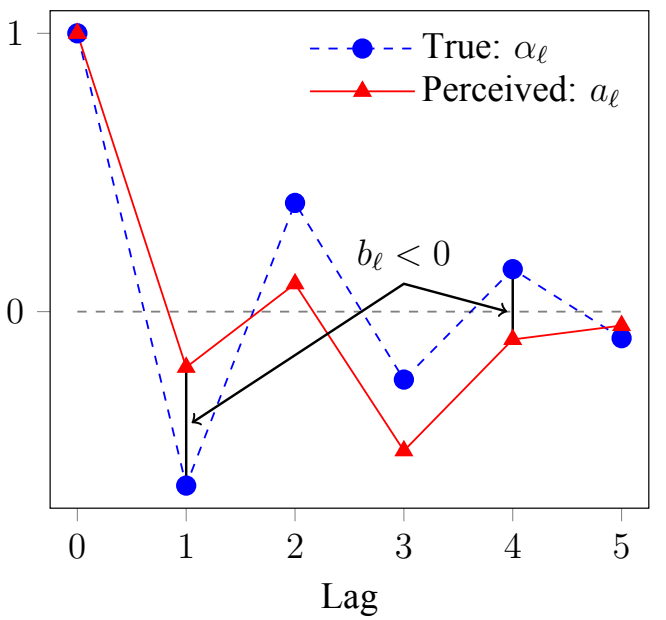

(b) Negative autocorrelation

Figure 1: Measurement framework illustrated. The dashed blue line shows the true impulse response function (IRF) of an AR(1) process: $x_{t}=\rho x_{t-1}+\varepsilon_{t}$ with $\rho \in(-1,1)$. The solid red line plots the IRF of the process as it may be perceived by the agent (an example). Left panel: Positively autocorrelated process $(\rho>0)$. Right panel: Negatively autocorrelated process $(\rho<0)$.

of the true process for $x_{t}$ (not the forecast errors). The solid red line plots an example IRF of how the process may be perceived by the agent. As seen in the picture, the bias coefficients $b_{\ell}$ are equal to the difference between the two IRFs. Our specification of expectations is flexible enough to allow for both overreaction and underreaction at different lags. In the case of positively autocorrelated $x_{t}$ (shown in the left panel) we have overreaction for $\ell \in\{1,2\}$ and underreaction for $\ell \geq 3$. Since the forecast errors are just the difference between the realized value and the forecast, the bias coefficients are equal to the IRF of forecast errors.

The right panel of Figure 1 makes it clear why we multiply the bias coefficients by $\operatorname{sgn}\left(\alpha_{\ell}\right)$. We interpret overreaction to mean that the perceived impulse response is larger than the true impulse response in absolute value. Multiplying by $\operatorname{sgn}\left(\alpha_{\ell}\right)$ ensures that a positive bias coefficient indicates overreaction when the true impulse response is negative. For $\ell=3$, for example, the perceived impulse response is smaller than the true impulse response, but larger in absolute value, and we classify this bias as overreaction. The symmetric opposite case is given by $\ell=1$. 


\subsection{Estimation}

If we could observe the true shocks (for example, in an experimental setting), ${ }^{9}$ it would be straightforward to estimate the bias coefficients by simply regressing forecast errors $e_{t}=x_{t}-\mathbb{F}_{t-1}\left[x_{t}\right]$ on past shocks: ${ }^{10}$

$$
e_{t}=\alpha+\beta_{0} \varepsilon_{t}+\beta_{1} \varepsilon_{t-1}+\cdots+\beta_{K} \varepsilon_{t-K}+u_{t} .
$$

The estimated bias coefficients are then $\hat{b}_{\ell}=-\operatorname{sgn}\left(\alpha_{\ell}\right) \hat{\beta}_{\ell}$ for $\ell \geq 1$. As we discuss in Section 2.4, this approach is followed in several prominent existing studies, sometimes implicitly.

In many cases of interest, however, we do not have enough information to estimate the true shocks with much confidence. In these situations we can still recover the bias coefficients by estimating the IRF of the forecast errors. To estimate the IRF flexibly, we use the method of local projections (Jordá, 2005). For each $s=0,1, \ldots,(L-1)$, we estimate the following regression by least squares:

$$
e_{t+s}=\alpha^{(s)}+\beta_{1}^{(s)} e_{t-1}+\beta_{2}^{(s)} e_{t-2}+\cdots+\beta_{K}^{(s)} e_{t-K}+u_{t}
$$

Here, $K$ denotes the number of lagged forecast errors included in the local projection. The estimated bias coefficients (for $\ell \geq 1$ ) are then $\hat{b}_{\ell}=-\operatorname{sgn}\left(\alpha_{\ell}\right) \hat{\beta}_{1}^{(\ell-1)}$. The timeinvariant bias coefficient $b_{0}$ is estimated by simply calculating the sample average of $e_{t}$.

While our preferred estimation method is local projections, it is possible to estimate the bias coefficients by fitting a high-order moving average model using maximum likelihood. In our experience, when maximum likelihood estimation works numerically, the resulting point estimates are very similar; an example is provided in Section 6. However, there are major benefits to using local projections. First, it is immediate to extend our method to cases in which we have multiple forecasters or want to pool multiple fore-

\footnotetext{
9 There is a large experimental literature on expectations that dates back to at least Schmalensee (1976). Schmalensee cites Fisher (1962) as a source for his experimental design, but the reference by Fisher is difficult to obtain. A recent experimental study that is particularly relevant for the present paper is Ma, Landier, and Thesmar (2017). Ma, Landier, and Thesmar estimate an empirical model of expectation formation; they also show how expectations respond to a unit shock in the model they consider (their Figure 5).

${ }^{10}$ An alternative approach would regress ex-ante forecast errors on past shocks. This approach, however, imposes stronger informational requirements. In some situations, we may be able to observe some of the true shocks even if we do not know the true conditional expectation $\mathbb{E}_{t-1}\left[x_{t}\right]$. For example, suppose that $\varepsilon_{t}=\varepsilon_{t}^{(1)}+\varepsilon_{t}^{(2)}$ where $\varepsilon_{t}^{(1)}$ and $\varepsilon_{t}^{(2)}$ are independent of each other, but we only observe $\varepsilon_{t}^{(1)}$. Then, we could estimate the regression in Eq. (5) consistently by using $\varepsilon_{t}^{(1)}$ in place of $\varepsilon_{t}$, even though the ex-ante forecast error is unknown.
} 
casts (panel data). Second, it is straightforward to adjust the standard errors for various forms of clustering. ${ }^{11}$ Finally, maximum likelihood estimation of high-order moving average models can be numerically challenging.

Intuition. Some readers may still wonder how we can recover the bias coefficients if the $\varepsilon_{t}$ 's in Eq. (4) are unobserved. The bias coefficients are identified from the structure of autocorrelation in the forecast errors. Suppose that the true process for $x_{t}$ is positively autocorrelated but the agent underreacts to new information. In that case, the forecast errors will be positively autocorrelated. With overreaction, in contrast, we would see negatively autocorrelated forecast errors. Implicitly, the method in Eq. (6) maps the observed autocorrelations into an implied IRF.

To see this in more detail, suppose that $x_{t}$ follows an AR(1) process $x_{t}=\rho x_{t-1}+\varepsilon_{t}$, $\rho \in(-1,1)$, and consider the following two models of expectations, one exhibiting underreaction and one exhibiting overreaction. (The precise descriptions of these models are given in Section 3.) For the sticky information model proposed by Mankiw and Reis (2002), forecast errors turn out to also follow an AR(1) process (see Section 3.2):

$$
e_{t}=\lambda \rho e_{t-1}+\varepsilon_{t}
$$

Here $\lambda \in[0,1]$ is a parameter measuring the stickiness of expectations. If the process is positively autocorrelated $(\rho>0)$, forecast errors are also positively autocorrelated. The IRF of forecast errors decays geometrically.

Now, in contrast, suppose that expectations are diagnostic (Bordalo, Gennaioli, and Shleifer, 2018). In this case, forecast errors follow an MA(1) process (see Section 3.3):

$$
e_{t}=\varepsilon_{t}-\theta \rho \varepsilon_{t-1}
$$

where $\theta \geq 0$ is a parameter capturing the extent to which agents overweight representative events. Therefore, forecast errors are negatively correlated at the first lag. At all other lags, forecast errors are uncorrelated. The IRF of forecast errors is equal to $(-\theta \rho)$ at the first lag and zero at all subsequent lags.

\subsection{Generalization}

While the assumptions used to develop our basic framework in Section 2.1 are relatively weak, they may not be tenable in all applications. In particular, we assumed that $x_{t}$ is

\footnotetext{
${ }^{11}$ For example, when we have multiple forecasters forecasting the same variable, it is essential to account for the cross-sectional correlation between the forecast errors of individual forecasters, as pointed out by Keane and Runkle (1990).
} 
weakly stationary, and that subjective expectations can be written as an infinite sum of the same Wold shocks that are driving $x_{t}$.

Thankfully, the framework can be generalized significantly by directly considering the IRF of the ex-post forecast errors. Economically, the IRF of the forecast errors represents the difference between the true IRF of $x_{t}$ and the IRF of $x_{t}$ as it is perceived by the agents. Hence, the IRF of the forecast errors can be naturally interpreted as a measure of under- or overreaction even when some of the assumptions in Section 2.1 are violated.

Formally, following Jordá (2005, Eq. (1)), define the IRF of some variable $y_{t}$ as the difference between the two forecasts

$$
\operatorname{IRF}(t, s)=\hat{\mathbb{E}}\left[y_{t+s} \mid \varepsilon_{t}=1, \Omega_{t-1}\right]-\hat{\mathbb{E}}\left[y_{t+s} \mid \varepsilon_{t}=0, \Omega_{t-1}\right], s=0,1,2, \ldots
$$

where $\hat{\mathbb{E}}$ denotes the optimal mean-squared error prediction, and the information set $\Omega_{t-1}$ equals $\left(y_{t-1}, y_{t-2}, \ldots\right)^{\top} .{ }^{12} \mathrm{We}$ can then write the IRF of forecast errors as

$$
\begin{gathered}
\operatorname{IRF}(t, s)=\underbrace{\left\{\hat{\mathbb{E}}\left[x_{t+s} \mid \varepsilon_{t}=1\right]-\hat{\mathbb{E}}\left[x_{t+s} \mid \varepsilon_{t}=0\right]\right\}}_{\text {actual impulse response }} \\
-\underbrace{\left\{\hat{\mathbb{E}}\left[\mathbb{F}_{t+s-1}\left(x_{t+s}\right) \mid \varepsilon_{t}=1\right]-\hat{\mathbb{E}}\left[\mathbb{F}_{t+s-1}\left(x_{t+s}\right) \mid \varepsilon_{t}=0\right]\right\}}_{\text {perceived impulse response }} .
\end{gathered}
$$

Hence, as long as Eq. (6) yields consistent estimates of the IRF of forecast errors, the estimated coefficients are economically meaningful as measures of under- or overreaction. ${ }^{13}$

This observation can be used to calculate the bias coefficients even when subjective expectations cannot be written as a linear combination of the same Wold shocks that are driving $x_{t}$. Provided that the forecast errors are weakly stationary-a much weaker assumption than what was considered in Section 2.1-we can write the forecast errors as

$$
e_{t}=-b_{0}+\sum_{\ell=0}^{+\infty} \theta_{\ell} \xi_{t-\ell}
$$

for some square-summable coefficients $\theta_{\ell}$ and a white noise series $\xi_{t}$. Then, we can define the bias coefficients much in the same way as in Section 2.1. We provide an example of such a calculation in Appendix A (Section A.6).

\footnotetext{
${ }^{12}$ See also Koop, Pesaran, and Potter (1996, Eq. (3)). Koop, Pesaran and Potter define their generalized impulse response function similarly, except that they do not condition on $\varepsilon_{t}=0$ in the second conditional expectation.

${ }^{13}$ See Jordá (2005, pp. 164-165) for a discussion of when local projections recover the true IRF.
} 


\subsection{Related Work}

We now discuss how our framework relates to existing work.

The paper that introduced rational expectations (Muth, 1961) already had a section on "Deviations from Rationality" (p. 321). In that section Muth studied a specification of expectations (his equation 3.18) that is a special case of our Eq. (2). In the notation of the present model, Muth allowed the subjective reaction to current news $\left(a_{1}\right)$ to differ from the true reaction of the process $\left(\alpha_{1}\right)$. In the present paper we continue in Muth's steps by allowing the subjective reaction to news in all periods to be different from the true reaction of the process. In addition, we show how to estimate the bias coefficients empirically. ${ }^{14}$

Broadly speaking, existing empirical approaches to measuring biases in expectation formation tend to impose assumptions on either (i) the underlying process; or (ii) the way expectations are formed. In the context of our framework, these assumptions correspond to Eqs. (1) and (2). ${ }^{15}$

In category (i), this paper is most closely related to Coibion and Gorodnichenko (2012). Similarly to our work, they study how forecast errors respond to shocks. While the authors do not frame their theoretical discussion in terms of bias coefficients, they derive how the forecast errors react to shocks in a number of models of expectations. These calculations are the same as those that we perform in our paper. However, in their empirical work Coibion and Gorodnichenko directly estimate the shocks using methods that are somewhat specific to their setting (expectations of macroeconomic variables). Our method has weaker informational requirements and may be more portable across applications. Nevertheless, when the restrictions made to estimate the shocks are in fact satisfied, the procedure employed by Coibion and Gorodnichenko typically yields more precise estimates (see Section 5). ${ }^{16}$

A prominent literature on expectations of stock returns also falls into category (i).

\footnotetext{
${ }^{14}$ Our specification of expectations in Eq. (2) is equivalent to the specification of "extrapolative models" in Pesaran and Weale (2006, Eq. (9)) who specify expectations as an infinite linear combination of past realizations of $x_{t}$. Applying the Wold's Representation Theorem to past values of $x_{t}$ in the specification by Pesaran and Weale would yield the same specification that is used in our paper.

${ }^{15}$ There is also a large literature that tests rational expectations without imposing such assumptions; see Pesaran and Weale (2006, Section 5). However, results from these tests are not necessarily straightforward to interpret without additional structure, as observed by Coibion and Gorodnichenko (2015, p. 2651): “[...] when traditional tests identify a rejection of the null hypothesis of FIRE [full information rational expectations], this rejection is not directly informative about other theories of the expectations formation process in the absence of a clear theoretical mapping from the theory to the empirical tests."

${ }^{16}$ An advantage of our method is that it uses all of the variation in $x_{t}$. In contrast, it may only be possible to measure a fraction of the variation in the true shocks in practice. If only a small fraction of the variation in the true shocks is used to apply the Coibion-Gorodnichenko procedure, our method may well lead to more precise estimates.
} 
At short horizons, classic asset pricing theory predicts that stock prices should follow martingales, and therefore changes in stock prices should be unpredictable (see, e.g. Cochrane, 2005, p. 22). Hence, a reasonable first approximation for stock returns is that $x_{t}=\varepsilon_{t}$, where $\varepsilon_{t}$ is a martingale difference sequence. In that case, the IRF representation in Eq. (4) implies that

$$
\mathbb{F}_{t-1}\left[x_{t}\right]=b_{0}+\sum_{\ell=1}^{+\infty} b_{\ell} x_{t-\ell}
$$

As a result, the bias coefficients can be recovered by simply regressing current expectations on past stock returns. The procedure is likely to yield more precise estimates than our method. Extrapolative expectations of stock returns have been documented by Graham and Harvey (2001), Vissing-Jorgensen (2004), Dominitz and Manski (2011) and Greenwood and Shleifer (2014), among others, and often interpreted as prima facie evidence for overreaction. Our framework highlights that stock returns must be uncorrelated over the relevant time horizon for the above specification to yield a meaningful measure of overreaction. If stock returns are correlated over time, some degree of extrapolation is justified. ${ }^{17}$

An important example of a category (ii) paper is given by Coibion and Gorodnichenko (2015). In that paper, the authors show that regressing forecast errors on lagged forecast revisions recovers structural parameters in a number of models featuring underreaction. Importantly, it is not necessary to know the data-generating process to recover parameters governing expectation formation. However, making the link between empirical estimates and structural parameters requires assumptions about how expectations are formed. If these assumptions are violated, it may not be straightforward to interpret the empirical estimates, analogously to the stock market case discussed above. Once again, if the assumptions are in fact satisfied, the method typically yields more efficient estimates than our procedure.

\section{Mapping Existing Models}

We now show how existing models of expectations can be mapped into our framework. The exercise leads to two key takeaways. First, our framework is flexible enough to nest all major models of expectations as special cases. Second, the implied bias coefficients provide a useful lens for looking at models of expectations. For instance, some models

\footnotetext{
${ }^{17}$ Cutler, Poterba, and Summers (1990a) document that in a sample of 13 developed countries over 19601988 , the autocorrelation of yearly excess aggregate stock market returns is roughly 0.02 (s.e. $=0.004$ ).
} 
that may intuitively be thought to only exhibit underreaction, in fact, imply overreaction at some lags.

To obtain closed-form expressions, we assume that $x_{t}$ follows a stationary $\operatorname{AR}(1)$ :

$$
x_{t}=\rho x_{t-1}+\varepsilon_{t}, \rho \in(-1,1),
$$

where $\varepsilon_{t}$ is a white noise error term. The simple AR(1) process is a reasonable first approximation for many economic time series. For more complicated processes, it is often difficult to obtain the bias coefficients analytically. In those cases, however, it is straightforward to obtain estimates by simulation.

To streamline the exposition, we discuss four major models of expectations in the main text (rational expectations, sticky information, diagnostic expectations, and adjustment costs). In Appendix A, we show how to obtain bias coefficients for models of adaptive expectations, noisy information, misperceived law of motion, extrapolative expectations, learning, and overconfidence.

The results for the models discussed in the current section are summarized in Figure 2. As shown in the figure, existing models have sharp predictions on the structure of bias coefficients. The sticky information model by Mankiw and Reis (2002) implies that the bias coefficients are negative and decay geometrically (dashed blue line). In contrast, diagnostic expectations of Bordalo, Gennaioli, and Shleifer (2018) predict that the agent overreacts to current news but reacts rationally to all past news (dotted magenta line). Finally, we plot the bias coefficients for a model in which the agent is rational but must incur a quadratic adjustment cost to change forecasts from one period to the next (solid red line). These adjustment costs may be interpreted as representing career concerns (in an admittedly reduced-form fashion). At the chosen parameter values, the model with adjustment costs predicts strong underreaction to current news but mild overreaction to news received further in the past. The result illustrates how the bias coefficients can be helpful in understanding models of expectations.

\subsection{Rational Expectations}

We now show how to map four specific models into our framework (see Appendix A for additional models). Rational expectations in the sense of Muth (1961) are given by

$$
\begin{aligned}
& a_{\ell}=\rho^{\ell} \\
& b_{\ell}=0
\end{aligned}
$$




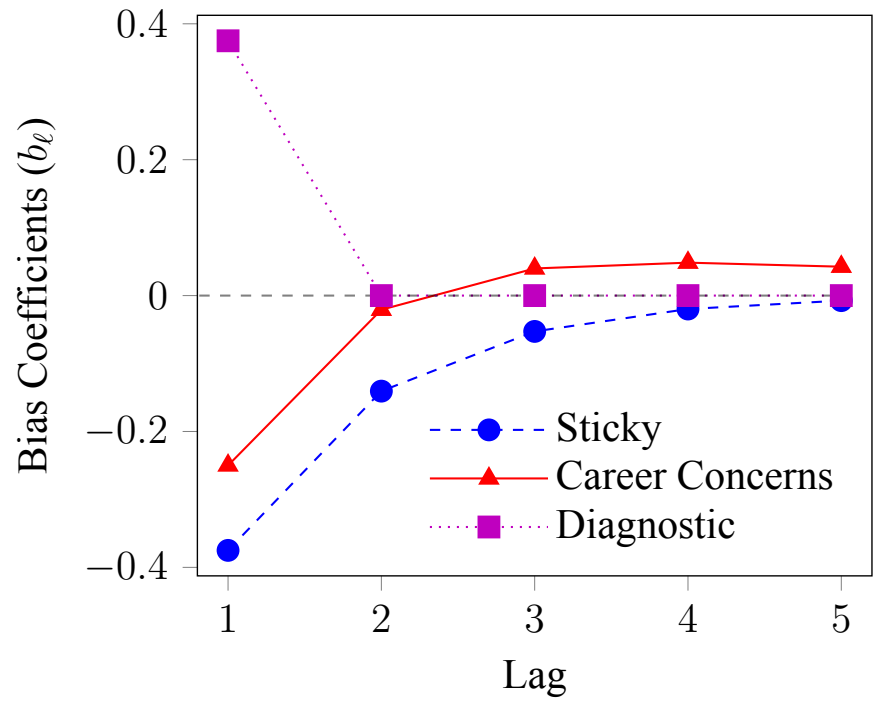

Figure 2: Bias coefficients for selected models of expectations. Positive bias coefficients indicate overreaction to news at a particular lag, and negative coefficients indicate underreaction. Unbiased reaction to news is given by a zero bias coefficient. The underlying process for $x_{t}$ is $x_{t}=0.75 x_{t-1}+\varepsilon_{t}$. The models shown are: (i) sticky information model of Mankiw and Reis (2002) with $\lambda=0.50$; (ii) forecasting with adjustment costs (Section 3.4) with $\alpha=1.0$ and $\delta=0.50$; and (iii) diagnostic expectations of Bordalo, Gennaioli, and Shleifer (2018) with $\theta=0.50$.

Here, the perceived response to a shock, $a_{\ell}$, is identical to the true response of $x_{t}$. As a result, all bias coefficients are zero.

\subsection{Sticky Information}

Consider the sticky information model of expectations proposed by Mankiw and Reis (2002); see also Carroll (2003). Each period a fraction $(1-\lambda) \in(0,1]$ of agents update their forecast to the full-information rational expectation. The remaining agents use information obtained in some previous period to form expectations that are rational conditional on their information set. ${ }^{18}$ Given these assumptions, expectations at the aggregate (or consensus) level follow

$$
\mathbb{F}_{t}\left[x_{t+1}\right]=(1-\lambda) \sum_{\ell=0}^{+\infty} \lambda^{\ell} \mathbb{E}_{t-\ell}\left[x_{t+1}\right]
$$

For the AR(1) model, we have that $\mathbb{E}_{t-\ell}\left[x_{t+1}\right]=\rho^{\ell+1} x_{t-\ell}$, and some algebra yields

$$
\mathbb{F}_{t}\left[x_{t+1}\right]=\sum_{\ell=0}^{+\infty} \rho^{\ell+1}\left(1-\lambda^{\ell+1}\right) \varepsilon_{t-\ell} .
$$

\footnotetext{
${ }^{18}$ Reis (2006, Section 5) provides a microfoundation for the Poisson adjustment process.
} 
As a result, we find that

$$
\begin{aligned}
& a_{\ell}=\rho^{\ell}\left(1-\lambda^{\ell}\right) \\
& b_{\ell}=-\operatorname{sgn}\left(\rho^{\ell}\right)(\lambda \rho)^{\ell}
\end{aligned}
$$

As long as expectations do not adjust to news immediately $(\lambda>0)$, the sticky information model exhibits underreaction at all lags. ${ }^{19}$

As seen above, the bias coefficients depend on both (i) how people form expectations; and (ii) the data-generating process. Different processes for $x_{t}$ will imply different bias coefficients, even if people form expectations in the same way. In the present example, the bias coefficients are larger in absolute value if the process is more persistent. Intuitively, underreaction is more severe when the process is highly persistent.

\subsection{Diagnostic Expectations}

Suppose that the agent has diagnostic expectations as in Bordalo, Gennaioli, and Shleifer (2018) and overweights representative events. Bordalo, Gennaioli, and Shleifer (2018, Proposition 1) show that in this case expectations follow

$$
\mathbb{F}_{t}\left[x_{t+1}\right]=\mathbb{E}_{t}\left[x_{t+1}\right]+\theta\left\{\mathbb{E}_{t}\left[x_{t+1}\right]-\mathbb{E}_{t-1}\left[x_{t+1}\right]\right\}, \theta \geq 0
$$

where $\theta$ is a parameter capturing the extent to which the agent overweights representative events. The expression can be rewritten as $\mathbb{F}_{t}\left[x_{t+1}\right]=\mathbb{E}_{t}\left[x_{t+1}\right]+\rho \theta \varepsilon_{t}$. Therefore, diagnostic expectations imply that

$$
a_{\ell}=\left\{\begin{array}{ll}
\rho(1+\theta) & \text { if } \ell=1 \\
\rho^{\ell} & \text { if } \ell \geq 2
\end{array} \text { and } b_{\ell}= \begin{cases}\theta|\rho| & \text { if } \ell=1 \\
0 & \text { if } \ell \geq 2\end{cases}\right.
$$

Hence, diagnostic expectations predict overreaction to current news and unbiased reaction to all other news. ${ }^{20}$

\footnotetext{
${ }^{19}$ Underreaction at all lags with sticky information extends to more general processes. To see this, consider a general $x_{t}$ with a Wold representation as in Eq. (1) and perform the same calculations as for the $\mathrm{AR}(1)$ case. The calculation shows that in the general case $b_{\ell}=-\operatorname{sgn}\left(\alpha_{\ell}\right) \lambda^{\ell} \alpha_{\ell}$.

${ }^{20}$ The prediction is conditional on the underlying process being an AR(1). A distinctive feature of diagnostic expectations is that they depend on the structure of the underlying process, i.e., Eq. (9) is endogenous to the model. In the sticky information model, parameter $\lambda$ is also likely to be endogenous to the process.
} 


\subsection{Adjustment Costs}

Finally, we assume the agent has rational expectations but faces a cost in adjusting forecasts from one period to the next. We interpret the adjustment cost as a stand-in for reputational costs or career concerns. For example, forecasters who change their forecasts by large amounts may be perceived as having lower forecasting ability.

Similarly to Coibion and Gorodnichenko (2015, p. 2660), suppose that in each period $t$ the agent makes a forecast of $x_{t+1}$. The agent wishes to minimize the mean-squared error of the prediction but faces a quadratic adjustment cost. ${ }^{21}$ Denoting the current value of $x_{t}$ as $x$ and the current forecast by $F^{\prime}$, the Bellman equation of the agent is given by

$$
V(x, F)=\min _{F^{\prime}} \frac{1}{2} \mathbb{E}\left[\left(\rho x+\tilde{\varepsilon}-F^{\prime}\right)^{2}\right]+\frac{\alpha}{2}\left(F^{\prime}-F\right)^{2}+\delta \mathbb{E}\left[V\left(\rho x+\tilde{\varepsilon}, F^{\prime}\right)\right],
$$

where $\alpha \geq 0$ is the weight on the adjustment cost, $\delta \in(0,1)$ is a discount factor, and we have used tildes to denote random variables. The first-order condition is

$$
-\left(\rho x-F^{\prime}\right)+\alpha\left(F^{\prime}-F\right)+\delta \mathbb{E}\left[V_{F}\left(\rho x+\tilde{\varepsilon}, F^{\prime}\right)\right]=0 .
$$

The envelope condition is just $V_{F}(x, F)=-\alpha\left(F^{\prime}-F\right)$. Therefore, the optimal forecast is given by

$$
\left(F^{\prime}\right)^{*}=\frac{\rho x+\alpha(1-\delta) F}{1+\alpha(1-\delta)} .
$$

If the agent is fully patient $(\delta=1)$ or there is no adjustment $\operatorname{cost}(\alpha=0)$, the forecast coincides with the true conditional expectation. In the other extreme, if $\alpha \rightarrow+\infty$, then it is optimal to never change the forecast.

Defining $\phi \equiv \alpha(1-\delta) /[1+\alpha(1-\delta)]$, the optimal forecasting rule is

$$
\mathbb{F}_{t}\left[x_{t+1}\right]=(1-\phi) \rho x_{t}+\phi \mathbb{F}_{t-1}\left[x_{t}\right]
$$

Performing similar manipulations to those in Appendix A.1, we arrive at

$$
\mathbb{F}_{t}\left[x_{t+1}\right]=(1-\phi) \rho \sum_{\ell=0}^{+\infty}\left[\frac{\phi^{\ell+1}-\rho^{\ell+1}}{\phi-\rho}\right] \varepsilon_{t-\ell} .
$$

\footnotetext{
${ }^{21}$ The setup is different from a situation in which an agent does not want to deviate from the consensus forecast, leading to game-theoretic considerations. This alternative situation is fully characterized by Coibion and Gorodnichenko (2012, pp. 126-129) who show-similarly to the model with sticky information - that the forecast errors follow an AR(1) process, implying geometrically decaying bias coefficients.
} 
Hence, the bias coefficients are equal to

$$
\begin{aligned}
& a_{\ell}=(1-\phi) \rho\left[\frac{\phi^{\ell}-\rho^{\ell}}{\phi-\rho}\right] \\
& b_{\ell}=\operatorname{sgn}\left(\rho^{\ell}\right)\left(a_{\ell}-\rho^{\ell}\right)
\end{aligned}
$$

A first intuition may be that adjustment costs can only generate underreaction. However, inspecting the expressions above, it is clear that this is not the case. Indeed, the bias coefficients for this model in Figure 2 are positive for $\ell \geq 2$ at some parameter values. Hence, the model with adjustment costs may generate overreaction (to more distant news).

The example also shows that the first-order autocorrelation of forecast errors can be misleading as a measure of underreaction. For the present model, the first-order autocorrelation is equal to

$$
\operatorname{Corr}\left(e_{t}, e_{t-1}\right)=\frac{\phi \rho[1+\phi+\rho(1-\phi)]}{1+\rho+\phi(1-\rho)[1+\rho(1-\phi)]} .
$$

Since $\phi \in[0,1)$, if $x_{t}$ is positively autocorrelated, the first-order autocorrelation of forecast errors is always positive. Nevertheless, as already discussed, expectations may exhibit overreaction. Hence, the first-order autocorrelation can be misleading as a measure of underreaction. For example, at the parameter values used in Figure 2, the autocorrelation is equal to roughly 0.24 , even though expectations overreact to news in multiple periods. Ignoring potential overreaction in later periods, as is commonly done in existing empirical work, can lead to incorrect inferences.

\section{Extensions}

We now discuss a number of extensions to the basic framework, including multiple-stepahead forecasts, heterogeneity, measurement error, and forecasting multiple variables. Finally, we present an example in which expectations are biased but the implied bias coefficients are zero. The example illustrates that some types of bias may go undetected by our method.

\subsection{Multiple-Step-Ahead Forecasts}

Forecasters commonly make multiple-step-ahead forecasts. For instance, we may observe an analyst making one-year-ahead inflation forecasts every quarter. It is straightforward to modify our methodology to account for such cases. 
Suppose that the agent makes $h$-step ahead forecasts with $h \geq 1$ denoting the forecast horizon. As before, $x_{t}$ is given by Eq. (1), and we assume that the forecasts are generated as

$$
\mathbb{F}_{t}\left[x_{t+h}\right]=b_{0}+\sum_{\ell=0}^{+\infty} a_{\ell+h} \varepsilon_{t-\ell} .
$$

Performing the same calculations as in Section 2.1 shows that

$$
x_{t}-\mathbb{F}_{t-h}\left[x_{t}\right]=-b_{0}-\sum_{\ell=h}^{+\infty} \operatorname{sgn}\left(\alpha_{\ell}\right) b_{\ell} \varepsilon_{t-\ell}+\sum_{\ell=0}^{h-1} \alpha_{\ell} \varepsilon_{t-\ell} .
$$

Comparing the equation above to Eq. (4), we observe an additional term stemming from the multiple-step ahead nature of forecasts. Even if subjective expectations react to news in an unbiased way, the forecast errors are autocorrelated - up to lag $(h-1)$-if the underlying process is autocorrelated at these lags. The result is well known (see, e.g., Diebold and Lopez, 1996).

The methodology again boils down to estimating the IRF of the forecast errors. Differently from the one-step ahead case, the first $(h-1)$ impulse responses need to be discarded. The remaining impulse responses are converted to bias coefficients, with $b_{\ell+h}$ giving the biased reaction to news that arrived $\ell$ periods ago.

\subsection{Heterogeneity and Aggregation}

Existing research has documented that expectations are heterogeneous across individuals (e.g., Manski, 2004, Section 5). Our method can easily be applied to subsamples of the population. For example, we may estimate the bias coefficients for young and old forecasters. ${ }^{22}$ When the method is applied to the whole population, it recovers the average bias coefficients, as we now show.

Suppose that expectations of agent $i$ are given by

$$
\mathbb{F}_{i t}\left[x_{t+1}\right]=b_{i 0}+\sum_{\ell=0}^{+\infty} a_{i, \ell+1} \varepsilon_{t-\ell}, i=1,2, \ldots, N .
$$

Denote the consensus (average) forecast by $\mathbb{F}_{t}\left[x_{t+1}\right] \equiv \frac{1}{N} \sum_{i} \mathbb{F}_{i t}\left[x_{t+1}\right]$. Then, the fore-

\footnotetext{
${ }^{22}$ In principle, our method could be used to estimate individual-specific bias coefficients. In practice, however, that is infeasible because most existing datasets have few time series observations per each individual.
} 
cast error of the consensus forecast is equal to

$$
x_{t}-\mathbb{F}_{t-1}\left[x_{t}\right]=-\bar{b}_{0}-\sum_{\ell=1}^{+\infty} \operatorname{sgn}\left(\alpha_{\ell}\right) \bar{b}_{\ell} \varepsilon_{t-\ell}+\varepsilon_{t},
$$

where $b_{i \ell} \equiv \operatorname{sgn}\left(\alpha_{\ell}\right)\left(a_{i \ell}-\alpha_{\ell}\right)$ and $\bar{b}_{\ell} \equiv \frac{1}{N} \sum_{i} b_{i \ell}$. With heterogeneity, the bias coefficients of the consensus forecast reflect the average bias coefficients of the individual forecasts. Therefore, the estimated bias coefficients are likely to be similar irrespective of whether consensus or individual forecasts are used. We illustrate this result in our application to inflation forecasts in Section 6.

\subsection{Measurement Error}

In practice, we may only be able to measure subjective expectations with some error. Such error could arise because of imperfect measurement (e.g., survey questions may be confusing). Measurement error could also be given an economic interpretation if subjective expectations are inherently random. A concrete example of the latter is provided in Appendix A (Section A.6) that discusses a stylized model of overconfidence.

Measurement error tends to mask any existing predictability of the forecast errors, biasing the measured bias coefficients to zero. As a result, the empirically estimated bias coefficients are likely to represent a lower bound on the true bias coefficients. Measurement error is less of an issue in datasets in which many individual forecasts are available. For these datasets, averaging across forecasters reduces measurement error by virtue of the Law of Large Numbers (assuming that measurement errors across forecasters are not too dependent). ${ }^{23}$

In Appendix B.1 we provide explicit formulas for the attenuation bias caused by measurement error for two models of expectations (sticky information and diagnostic expectations). The attenuation bias can be substantial, underscoring the benefits of datasets with a large cross-sectional dimension.

While our method is sensitive to measurement error, so are most other existing techniques. For instance, a common test for the optimality of expectations with respect to some information set estimates

$$
x_{t}=\alpha+\beta \mathbb{F}_{t-1}\left[x_{t}\right]+u_{t}
$$

\footnotetext{
${ }^{23}$ Existing literature has developed techniques for consistently estimating time series models in the presence of measurement error, see Staudenmayer and Buonaccorsi (2005) and references therein. It may be possible to combine these techniques with the approach taken in our paper to obtain consistent estimates of the bias coefficients in the presence of measurement error. However, pursuing this path is outside the scope of the present paper.
} 
and then tests the joint restriction $\alpha=0$ and $\beta=1$. The test goes back to at least Mincer and Zarnowitz (1969, p. 9, who also credit Henri Theil, see p. 5); for a more detailed discussion, see Diebold and Lopez (1996). Clearly, even if $\alpha=0$ and $\beta=1$ in the equation above, if we only observe a noisy version of $\mathbb{F}_{t-1}\left[x_{t}\right]$, the estimate of $\beta$ will be biased towards zero. For the method of Coibion and Gorodnichenko (2015), measurement error can be even more pernicious and may even change the estimated sign, as shown in Appendix B.2.

\subsection{Multiple Variables}

We now consider how our method extends to the case of multiple variables.

First, we emphasize that the formulation in Section 2 does not presume that $x_{t}$ is generated by a univariate process, nor that agents are only using past values of $x_{t}$ in forming their expectations. As long as $x_{t}$ is covariance stationary, it has a Wold representation and can be written in the form given in Eq. (1). Hence, the substantive economic assumption is that expectations can be written as a linear combination of the same Wold shocks that are driving $x_{t}$. These shocks, in turn, may combine information about multiple economic variables. That being said, the method provided in Section 2 cannot answer some questions that may be of interest. For instance, if we find that people underreact to information about inflation, the method cannot say whether people underreact to news about monetary policy or developments in the labor market. Additional assumptions are necessary to answer these types of questions, as we now show.

Consider a mean-zero vector stochastic process $\mathbf{x}_{t}=\left(x_{1 t}, x_{2 t}, \cdots, x_{N t}\right)^{\top}$ that does not have any deterministic component. If $\mathbf{x}_{t}$ is covariance stationary, then the multivariate Wold's Representation Theorem (see, e.g., Hannan, 1970, Theorem 2, p. 158) implies that the process can be written as

$$
\mathbf{x}_{t}=\sum_{\ell=0}^{+\infty} \Lambda_{\ell} \varepsilon_{t-\ell}
$$

where $\varepsilon_{t} \equiv\left(\varepsilon_{1 t}, \varepsilon_{2 t}, \cdots, \varepsilon_{N t}\right)^{\top}$ is vector white noise, and $\boldsymbol{\Lambda}_{\ell}$ 's are $(N \times N)$ matrices.

Without loss of generality, suppose that the agent is forecasting the first $M \geq 1$ variables contained in $\mathbf{x}_{t}$, denoted by $\mathbf{x}_{t}^{f}$. We assume that the expectations of the agent can be written as

$$
\mathbb{F}_{t}\left[\mathbf{x}_{t+1}^{f}\right]=\mathbf{b}+\sum_{\ell=0}^{+\infty} \mathbf{A}_{\ell+1}^{f} \varepsilon_{t-\ell}
$$

where $\mathbf{A}_{\ell}^{f}$ 's are $(M \times N)$ matrices, and $\mathbf{b}$ is a $(M \times 1)$ vector capturing time-invariant 
bias.

Let $\Lambda_{\ell}^{f}$ denote the $(M \times N)$ matrix that is constructed by taking the first $M$ rows of $\Lambda_{\ell}$. We now extend our definition of under- and overreaction from Section 2 as follows. We say that the agent overreacts to shock $\varepsilon_{j, t-\ell}$ when forecasting $x_{i t}$ if the $i j$-th element of $\mathbf{A}_{\ell+1}$ is larger than the $i j$-th element of $\boldsymbol{\Lambda}_{\ell+1}$ in absolute value; underreaction is defined in a similar way. The key difference from the baseline setting is that with multiple shocks, the agent may simultaneously overreact to one piece information received in period $t$ and underrreact to a different piece of information received in that same period. We work out a concrete example of such simultaneous under- and overreaction in Appendix A (Section A.6).

Given the assumption above, the ex-post forecast errors are given by

$$
\mathbf{e}_{t}=-\mathbf{b}-\sum_{\ell=1}^{+\infty}\left(\mathbf{A}_{\ell}^{f}-\Lambda_{\ell}^{f}\right) \varepsilon_{t-\ell}+\varepsilon_{t}
$$

This equation generalizes Eq. (4) to the multivariate case. However, in contrast to Section 2, we can no longer recover the difference between $\mathbf{A}_{\ell}^{f}$ and $\Lambda_{\ell}^{f}$ by simply estimating the IRF of the forecast errors. For one thing, the matrix $\left(\mathbf{A}_{\ell}^{f}-\mathbf{\Lambda}_{\ell}^{f}\right)$ is of dimension $(M \times N)$, and so if $M<N$, we cannot hope to measure the reaction of the forecast errors to a shock in $\varepsilon_{j t}$ for a particular $j$. Even if we assume that the agent is forecasting all of the relevant variables $(M=N)$ and proceed to estimate the IRF of the forecast errors, the estimated coefficients only measure the response of forecast errors to reduced-form innovations. Economically, the IRF may not be of direct interest. For example, we may be interested to learn how inflation expectations respond to a monetary policy shock. To measure this response, as is well known, additional assumptions are necessary. ${ }^{24}$

\subsection{Inefficient Forecasts}

While our empirical procedure can capture rich forms of bias, there are some forms of bias that the method is unable to detect. In other words, there are situations in which expectations are biased in the sense of Section 2.1, and yet the implied bias coefficients are zero. In this section we provide one such example: If agents ignore information that is relevant for forecasting $x_{t}$, the loss in precision can go undetected by our procedure.

Suppose that $x_{t}$ has the Wold representation in Eq. (1). However, at time $t$ there is a public signal $s_{t}$ that is helpful for forecasting $\varepsilon_{t+1}$ with

$$
\varepsilon_{t+1}=s_{t}+\nu_{t+1}
$$

\footnotetext{
${ }^{24}$ For a recent discussion, see Ramey (2016).
} 
where $s_{t}$ and $\nu_{t+1}$ are mutually uncorrelated white noise series. Given the existence of the public signal, the true conditional expectation is equal to

$$
\mathbb{E}_{t-1}\left[x_{t}\right]=s_{t-1}+\sum_{\ell=1}^{+\infty} \alpha_{\ell} \varepsilon_{t-\ell},
$$

implying a forecast error of $\nu_{t}$. In contrast, if the forecaster correctly perceives that the true process is generated by Eq. (1) but ignores the public signal, the forecast will be biased (according to our definition in Section 2.1), with a forecast error of $\varepsilon_{t}$. Since $s_{t}$ and $\nu_{t+1}$ are uncorrelated, $\operatorname{Var}\left(\nu_{t}\right) \leq \operatorname{Var}\left(\varepsilon_{t}\right)$. Hence, ignoring the additional information in $s_{t}$ leads to less efficient forecasts. However, since $\varepsilon_{t}$ is white noise, the implied bias coefficients are zero. Of course, the example is quite special in that the signal is white noise. In more realistic cases, ignoring relevant information typically leads to non-zero bias coefficients (e.g., consider Section A.3 with $\hat{\rho}=0$ ).

\section{Monte Carlo Experiment}

To further investigate the validity of our methodology, we perform a Monte Carlo experiment. The purpose of the experiment is twofold. First, we gauge the statistical power of our procedure. Second, we investigate the performance of our method when some of the underlying assumptions are violated.

To facilitate comparison, the baseline design follows Coibion and Gorodnichenko (2012, Appendix B). The true process is an AR(1) calibrated to match the key features of quarterly GDP deflator inflation:

$$
x_{t}=\rho x_{t-1}+\varepsilon_{t} \text { with } \varepsilon_{t} \sim \text { i.i.d. } \mathcal{N}\left(0, \sigma_{\varepsilon}^{2}\right), t=1,2, \ldots, T .
$$

We set $\rho=0.85, \sigma_{\varepsilon}^{2}=1.005$ and use a sample size of $T=150$ periods. The value for $x_{1}$ is drawn from the stationary distribution of $x_{t}$, i.e., $\mathcal{N}\left(0, \sigma_{\varepsilon}^{2} /\left(1-\rho^{2}\right)\right)$.

Subjective expectations follow the sticky information model of Mankiw and Reis (2002):

$$
\mathbb{F}_{t}\left[x_{t+1}\right]=(1-\lambda) \rho x_{t}+\lambda \rho \mathbb{F}_{t-1}\left[x_{t}\right]^{25}
$$

The level of information stickiness is $\lambda=0.75$. We set $\mathbb{F}_{0}\left[x_{1}\right]=0$ to start up the recursion. To allow for measurement error, the observed forecasts, $\mathbb{F}_{t}^{*}\left[x_{t+1}\right]$, are equal

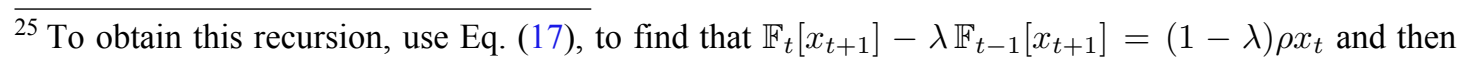
substitute $\mathbb{F}_{t-1}\left[x_{t+1}\right]=\rho \mathbb{F}_{t-1}\left[x_{t}\right]$ as derived in Appendix B.1 (Section B.2). 
to the true forecasts plus some noise $v_{t}$ (independent of the true shocks):

$$
\mathbb{F}_{t}^{*}\left[x_{t+1}\right]=\mathbb{F}_{t}\left[x_{t+1}\right]+v_{t}, v_{t} \sim \text { i.i.d. } \mathcal{N}\left(0, \sigma_{v}^{2}\right)
$$

As discussed in Section 4.3, measurement error could have multiple economic interpretations, including data collection problems (e.g., poorly constructed surveys) as well as expectations that are inherently random (e.g., overconfidence, see Section A.6).

We parametrize the size of measurement error by the $R^{2}$ of Eq. (10). From Eq. (8), the fraction of the variation in the observed forecasts that is explained by the true forecasts is

$$
R^{2}=\frac{\kappa \sigma_{\varepsilon}^{2}}{\kappa \sigma_{\varepsilon}^{2}+\sigma_{v}^{2}} \text { where } \kappa=\frac{(1-\lambda)^{2} \rho^{2}\left(1+\lambda \rho^{2}\right)}{\left(1-\rho^{2}\right)\left(1-\lambda \rho^{2}\right)\left(1-\lambda^{2} \rho^{2}\right)}
$$

In Appendix B.2, we show that the existing evidence on inflation expectations points to $\sigma_{v} \approx 0.21$, implying an $R^{2}$ of 0.95 at the chosen parameter values. Since other empirical settings may have more measurement error, we provide the results for a range of values for $R^{2}$. To avoid potential initial value effects, we simulate the model for 1,150 periods and discard the first 1,000 periods. The remaining 150 periods are then used for estimation.

The results are shown in Figure 3. The left column plots the estimated bias coefficients when the true shocks are observed. This estimation procedure regresses the forecast errors on the true shocks, as given in Eq. (5), and is essentially identical to the method proposed by Coibion and Gorodnichenko (2012). ${ }^{26}$ The top row has $R^{2}=1$ (no measurement error), and the lower rows include progressively more measurement error. The shaded areas give $90 \%$ of the Monte Carlo realizations.

The estimates obtained using the true shocks are unbiased. Measurement error does not introduce bias because noisy forecasts only lead to measurement error in the lefthand side of the regression. Measurement error only makes the estimates less precise.

The right column shows the estimates obtained using local projections, our preferred method when the true shocks are not observed. Local projections only use the forecast errors, as given in Eq. (6). With local projections, measurement error leads to an attenuation bias. As discussed in Section 4.3, this issue is not unique to our method. The bias can be substantial if measurement error is large. Perhaps more surprisingly, measurement error does not make the estimates less precise. The reason is that measurement error is present in both the left- and right-hand side variables of the regression. While measurement error in the left-hand side variable unambiguously makes least squares

\footnotetext{
${ }^{26}$ In their paper, Coibion and Gorodnichenko use a somewhat more parametric specification (see their Eq. (33)). In Appendix D, Coibion and Gorodnichenko consider a specification (their Eq. (D1)) that is equivalent to Eq. (5) and show that it yields similar results empirically.
} 

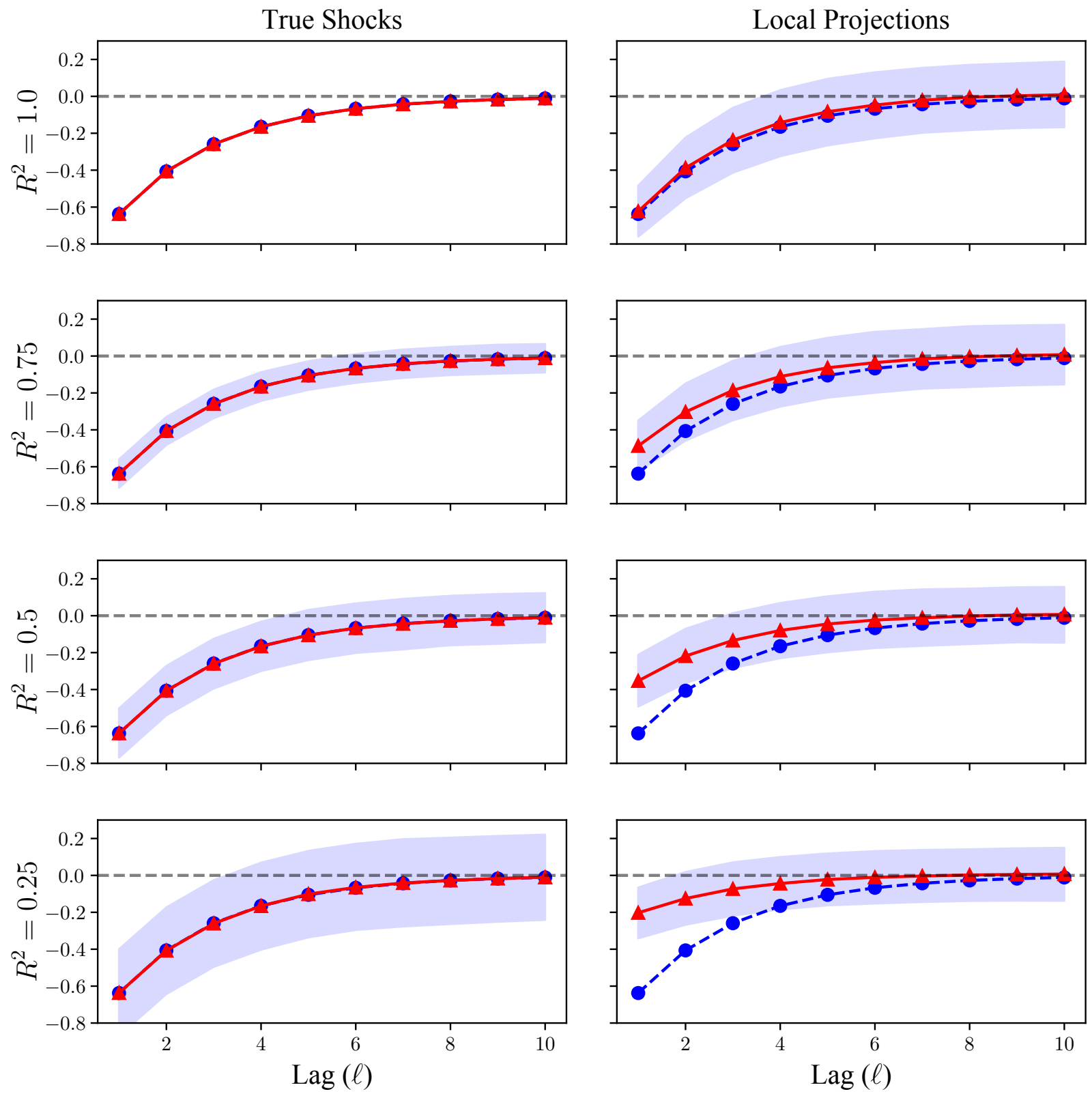

- -- True Bias Coefficients

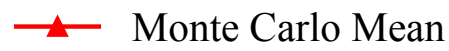

Figure 3: Baseline Monte Carlo results. The shaded areas give $90 \%$ of the Monte Carlo realizations $\left(5^{\text {th }}\right.$ and $95^{\text {th }}$ percentiles of the Monte Carlo realizations). 10,000 Monte Carlo replications are used for each figure. The top row has no measurement error; the lower rows have progressively more measurement error.

Left column: Bias coefficients estimated using the true shocks:

$$
x_{t}-\mathbb{F}_{t-1}\left[x_{t}\right]=\alpha+\beta_{0} \varepsilon_{t}+\beta_{1} \varepsilon_{t-1}+\cdots+\beta_{10} \varepsilon_{t-10}+u_{t} .
$$

Right column: Bias coefficients estimated using local projections (not using the true shocks):

$$
x_{t+s}-\mathbb{F}_{t+s-1}\left[x_{t+s}\right]=\alpha+\beta_{1}^{(s)}\left\{x_{t-1}-\mathbb{F}_{t-2}\left[x_{t-1}\right]\right\}+\cdots+\beta_{4}^{(s)}\left\{x_{t-4}-\mathbb{F}_{t-3}\left[x_{t-4}\right]\right\}+u_{t},
$$

where $s=0,1, \ldots, 9$. 
estimates less precise, measurement error on the right-hand side introduces variation that is helpful for identifying the value of the biased coefficient. We stress that local projections correctly identify the IRF of the observed forecast errors. The issue is that with measurement error, the IRF of the observed forecast errors is no longer the object of economic interest.

Comparing the two columns, we observe that our method has reasonable statistical power. For example, when $R^{2}=0.75$, our method is able to detect the first three bias coefficients in $90 \%$ of Monte Carlo simulations, whereas with true shocks, we can detect the first five bias coefficients. In addition, the comparison is not entirely fair since in practice we are unlikely to observe all of the shocks. As a result, it may be more reasonable to compare our method when $R^{2}=0.75$ to that of using the true shocks when $R^{2}=0.25$ or so. To be concrete, to apply their method, Coibion and Gorodnichenko (2012) first estimate various shocks to inflation. Coibion and Gorodnichenko show that technology shocks explain the largest share of the variation in inflation of all the shocks that they consider. However, even technology shocks only explain around $25 \%$ of the variation (their Table 2). In contrast, local projections directly use all of the variation in the variable that is being forecast.

Nevertheless, the results in Figure 3 imply that in some cases our method is unlikely to detect bias. For instance, consider expectations of stock market returns, as studied by Greenwood and Shleifer (2014), among others. If we assume that stock market returns are uncorrelated over time (as discussed in Section 2.4), then the estimates in Greenwood and Shleifer (2014, Table 3) suggest an upper bound for the bias coefficient at the first yearly lag of $0.03 .{ }^{27}$ Our method is unlikely to detect a bias of this magnitude. In the current simulation, our method fails to detect the bias coefficient at the fourth lag in many simulations, even when no measurement error is present. The magnitude of the bias coefficient at the fourth lag in the simulation is -0.165 , or roughly five times greater than the likely effect size in the stock market setting. This reasoning also suggests that there may be large efficiency gains from imposing additional structure when measuring overreaction in financial markets (such as imposing $\rho=0$ ).

In addition to the baseline simulation, we investigate the robustness of our methodology to a number of variations. We consider two changes to the underlying $x_{t}$ process. First, we suppose that $x_{t}$ is not covariance stationary and follows a random walk (i.e.,

${ }^{27}$ In Table 3, Greenwood and Shleifer regress expectations of stock market returns on realized stock market returns over the past year. For the case of Chief Financial Officers in the U.S. (column GH) who directly report a quantitative estimate of future stock returns, the estimated slope coefficient is 3.13 (s.e. $=1.25$ ). Greenwood and Shleifer measure expectations in percentages (e.g., 5 for an expected return of $5 \%$ ) whereas past stock returns are expressed as a fraction (e.g., 0.05 for a realized return of $5 \%$ ), see their Table 1. Dividing the slope coefficient by 100 leads to the number reported in the text. 
$x_{t}=x_{t-1}+\varepsilon_{t}$ and $x_{0}=0$ ), with all other parameters unchanged. Second, instead of normally distributed i.i.d. shocks, we suppose that the $\varepsilon_{t}$ 's follow a $\operatorname{GARCH}(1,1)$ :

$$
\begin{aligned}
\varepsilon_{t+1} & =\sigma_{t+1} z_{t+1}, z_{t} \sim \mathcal{N}(0,1) \\
\sigma_{t+1}^{2} & =\omega+\alpha \varepsilon_{t}^{2}+\beta \sigma_{t}^{2}
\end{aligned}
$$

To calibrate the process, we use the estimates for inflation reported by Capistrán and Timmerman (2009, Table 1): $\omega=0.02, \alpha=0.12$, and $\beta=0.86$. These parameter values imply an unconditional variance of 1 , which is very close to the unconditional variance of $\sigma_{\varepsilon}^{2}=1.005$ used in the baseline simulation. However, with a GARCH process, extreme realizations of $\varepsilon_{t}$ are much more likely.

Finally, we examine the robustness of the baseline simulation by examining an alternative model of expectations. Existing experimental work has documented that people's forecasts are often well approximated by simple linear functions of past forecasts and past values of $x_{t} \cdot{ }^{28}$ For that reason we also consider a simple model of extrapolative expectations:

$$
\mathbb{F}_{t}\left[x_{t+1}\right]=x_{t}+\gamma\left(x_{t}-x_{t-1}\right) .
$$

In a recent experiment on inflation forecasting, Pfajfar and Žakelj (2014) document that many people use such forecasting rules. Based on their findings, we pick $\gamma=0.50 .^{29}$

Finally, we consider a Markov-switching model of expectations. We assume that expectations follow Eq. (12) but with one change: Expectations can now be in one of two states. They can either be trend seeking with $\gamma_{H}=0.50$ or contrarian with $\gamma_{L}=-0.50$. The movement from the current $\gamma$ to the value of $\gamma$ in the next period, denoted by $\gamma^{\prime}$, is governed by a Markov chain. The transition matrix is symmetric with

$$
\begin{aligned}
& \mathbb{P}\left(\gamma^{\prime}=\gamma_{s} \mid \gamma=\gamma_{s}\right)=0.75 \\
& \mathbb{P}\left(\gamma^{\prime} \neq \gamma_{s} \mid \gamma=\gamma_{s}\right)=0.25
\end{aligned}
$$

where $s \in\{L, H\}$. While the particular numbers are less empirically grounded, the specification captures the fact-well documented in experiments-that people often switch between different internal models of expectations. ${ }^{30}$ Prominent theoretical mod-

\footnotetext{
${ }^{28}$ See, among many others, Hey (1994), Heemeijer, Hommes, Sonnemans, and Tuinstra (2009) and Bao, Hommes, and Makarewicz (2017).

${ }^{29}$ Pfajfar and Žakelj (2014, Table 5) find that for a plurality (but not a majority) of people, rational expectations cannot be rejected, and trend extrapolation is the second largest category of subjective expectations. While Pfajfar and Žakelj (2014) do not provide the average estimates for the trend extrapolation model, in Supplementary Material, they state that the estimated extrapolation coefficient lies between 0 and 1 in most cases. For that reason, we pick $\gamma=0.50$.

${ }^{30}$ See Pfajfar and Žakelj (2014) or Assenza, Bao, Hommes, and Massaro (2014).
} 

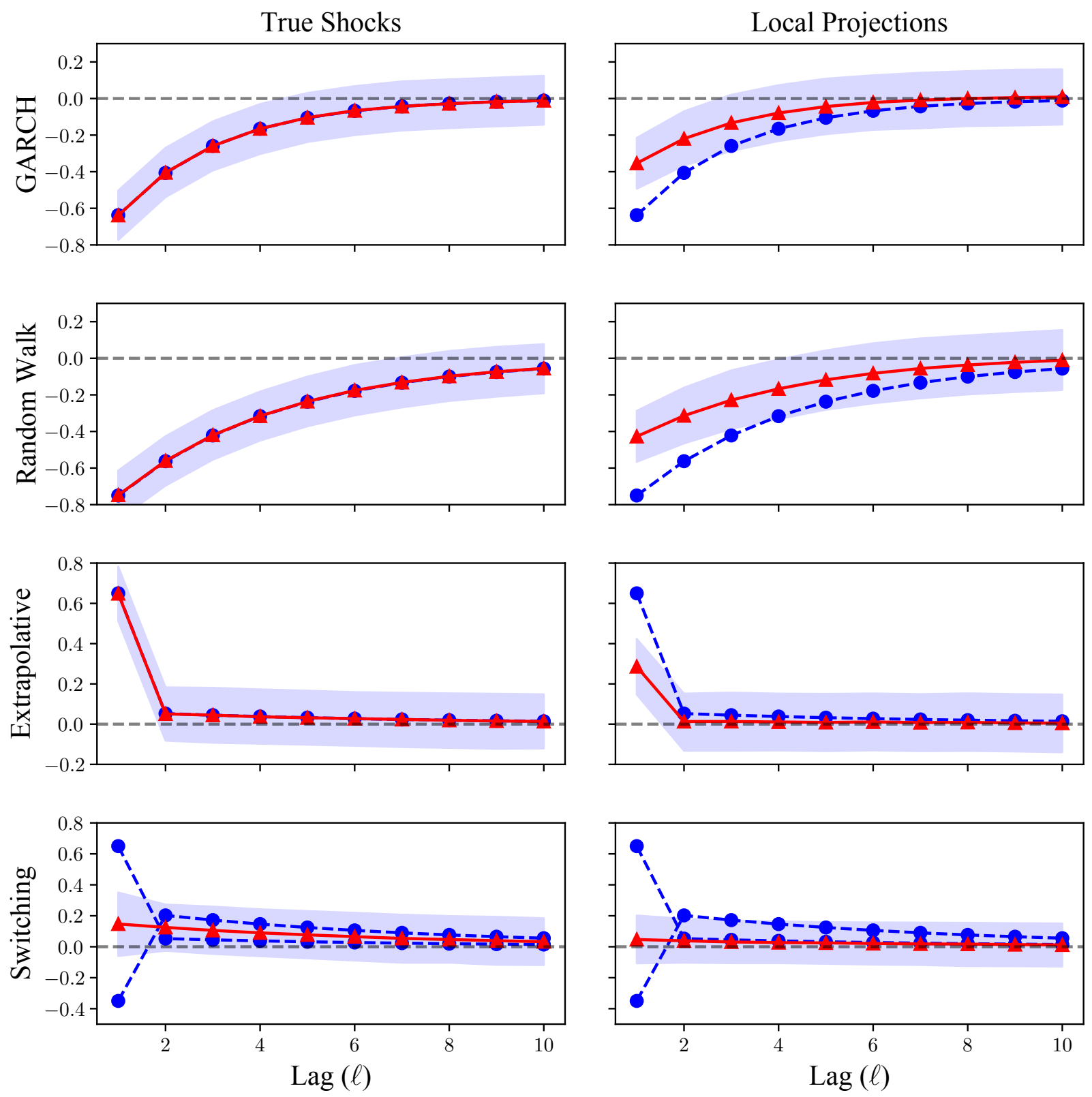

- -- True Bias Coefficients

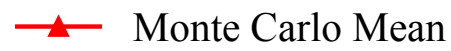

Figure 4: Additional Monte Carlo results. The shaded areas give $90 \%$ of the Monte Carlo realizations ( $5^{\text {th }}$ and $95^{\text {th }}$ percentiles of the Monte Carlo realizations). 10,000 Monte Carlo replications are used for each figure. The left column uses the true shocks to estimate bias coefficients; the right column uses local projections (without using the true shocks). The top row has no measurement error; the lower rows have progressively more measurement error. See Figure 3 for the exact regression specifications.

GARCH: $\operatorname{GARCH}(1,1)$ distributed shocks. Random Walk: random walk for the underlying process $(\rho=1)$. Extrapolative: extrapolative expectations with $\mathbb{F}_{t}\left[x_{t+1}\right]=x_{t}+\gamma\left(x_{t}-x_{t-1}\right)$ and $\gamma=0.50$. Switching: Markov-switching model with extrapolative expectations in which $\gamma=0.50$ or $\gamma=-0.50$ according to a Markov chain. The simulation with GARCH errors calibrates the value of $\sigma_{v}$ so that $R^{2}=0.50$ using Eq. (11). All other simulations use the same level of measurement error $\sigma_{v} \approx 0.963$ as in Figure 3 for $R^{2}=0.50$. 
els that feature switching expectations include Brock and Hommes (1997) and Barberis, Shleifer, and Vishny (1998). Our formulation is particularly motivated by the latter paper.

The results are shown in Figure 4. For the case of GARCH errors and a random walk for $x_{t}$, the results are very similar to those reported in the baseline setting for $R^{2}=0.50$. The findings for extrapolative expectations are also similar. While both local projections and the estimation procedure that uses the true shocks is able to detect the first bias coefficient, local projections are subject to an attenuation bias from measurement error. With Markov-switching expectations, although the true bias coefficients are not zero, both methods often fail to detect bias. The reason is simple: Expectations switch between two opposite types of bias. If the econometrician fails to notice the Markov-switching structure of expectations and estimates the bias coefficients using the full sample, the resulting estimates yield a time average of the true coefficients, which is close to zero. The example highlights how ignoring changes in expectations may significantly bias estimates.

\section{Application: Inflation Expectations}

We now turn to an empirical application of our method.

The application uses inflation forecasts from the Survey of Professional Forecasters (SPF). The SPF is currently run by the Federal Reserve Bank of Philadelphia. Each quarter, participants of the survey forecast a number of macroeconomic and financial variables. The names of the individual participants in the survey are not publicly known, limiting the scope for strategic considerations. The participants are professional forecasters; see Croushore (1993) for further discussion.

The dataset has been used extensively in prior work, with recent prominent studies including Carroll (2003), Mankiw, Reis, and Wolfers (2003), Coibion and Gorodnichenko (2012), and Coibion and Gorodnichenko (2015). ${ }^{31}$ As a result, the dataset provides a natural testing ground for our method. To streamline the discussion, we focus on the findings and explain how we construct the dataset in Appendix C.

\subsection{Bias Coefficients}

We study one-quarter ahead GDP deflator inflation forecasts. Summary statistics of the dataset are provided in Table $1 .{ }^{32}$ Both consensus (median) and individual-level fore-

\footnotetext{
31 The Philadelphia Fed provides an extensive list of studies that have used this dataset.

32 The number of participants in the SPF has not been constant over time. As a result, estimates using the consensus- and individual-level datasets implicitly weight the data somewhat differently. The
} 


\begin{tabular}{lrrrrrrrr}
\hline & $N$ & $\bar{x}$ & $\sigma_{x}$ & $\rho_{x}$ & $\bar{e}$ & $\mathrm{RMSE}$ & $\rho_{e}$ & $R_{\mathrm{adj}}^{2}$ \\
\hline Inflation: Consensus & 196 & 3.45 & 2.59 & 0.83 & -0.05 & 1.40 & 0.40 & 0.20 \\
Inflation: Individual & 7,475 & 3.80 & 2.67 & 0.81 & 0.02 & 1.96 & 0.32 & 0.16 \\
\hline
\end{tabular}

Table 1: Summary statistics. Root mean-squared error (RMSE) is calculated as $\sqrt{\frac{1}{N} \sum e_{t}^{2}}$ where $e_{t}$ is the forecast error. Persistence $\rho_{z}$ is measured by the estimate of $b$ in the regression $z_{t}=a+b z_{t-1}+v_{t}$. $R_{\text {adj }}^{2}$ is the adjusted $R$-squared in the regression of forecast errors on past four forecast errors $e_{t-1}, e_{t-2}, \ldots, e_{t-4}$

casts are considered. We construct the consensus forecasts from the individual forecasts available on the SPF website. To avoid bias stemming from data revisions, we use realtime data to measure the realized inflation.

The results are shown in Figure 5. The top panel plots the bias coefficients estimated using consensus forecasts. To calculate the bias coefficients, we first estimate the IRF of forecast errors using local projections, as in Eq. (6). Then, the estimated IRF is multiplied by $(-1)$ to obtain the bias coefficients. ${ }^{33}$ To be conservative, we show both the $65 \%$ and $95 \%$ confidence intervals calculated using Newey-West standard errors. We observe statistically significant negative bias coefficients for lag $1(p=0.005),{ }^{34}$ lag $3(p=0.022)$, and lag $4(p<0.001)$. The evidence suggests that participants in the SPF underreact to information that arrived up to one year ago. In Appendix D, we show that virtually identical results obtain — with somewhat smaller standard errorsif instead of local projections, we use maximum likelihood to fit a high-order moving average model (Figure 6).

The magnitude of underreaction is substantial. The point estimates indicate that a positive $1 \sigma$ shock to inflation in the current quarter leads the forecasters to underpredict inflation by roughly $0.30 \sigma$ four quarters from now.

The bottom panel of Figure 5 performs the same exercise using the individual-level forecasts. We estimate a panel-data equivalent of Eq. (6), including forecaster fixed effects. To account for the fact that the respondents are all forecasting the same variable, and the forecast errors may be correlated over time for a given respondent, we cluster

individual-level dataset implies a somewhat higher weight on observations coming from the earlier part of the sample. That is the reason why, for instance, the estimates of mean inflation differ across these two datasets.

${ }^{33}$ As shown in Appendix D (Figure 7), the IRF of inflation is positive at all the relevant lags, so that $\operatorname{sgn}\left(\alpha_{\ell}\right)=1$.

${ }^{34}$ At the time of responding to the survey, participants know the advance estimate of inflation in the previous quarter but not inflation in the current quarter (Federal Reserve Bank of Philadelphia, 2017, p. 21). As a result, interpretation of the bias coefficient in the first lag requires some care. On the one hand, forecasters have access to various real-time information on prices. On the other hand, they do not yet know the official number for inflation in the current quarter. In that sense, the one-step ahead forecast may really be a two-step ahead forecast. If that is the case, a non-zero bias coefficient at the first lag should not be interpreted as bias. See Keane and Runkle (1990) for further discussion. 


\begin{tabular}{lrl}
\hline Model & Parameter & SSR \\
\hline Misperception & 0.61 & 0.16 \\
Sticky & 0.51 & 0.18 \\
Adjustment Cost & 0.43 & 0.25 \\
Rational & $\mathrm{NA}$ & 0.32 \\
Diagnostic & 0.00 & 0.32 \\
Adaptive & 0.17 & 0.36 \\
Extrapolative & -0.31 & 0.55 \\
\hline
\end{tabular}

Table 2: Calibration exercise for matching the empirically estimated bias coefficients. The sum of squared residuals (SSR) is calculated as $\sum_{\ell=1}^{12}\left[\hat{b}_{\ell}-b_{\ell}\left(\theta^{*}\right)\right]^{2}$ where $\hat{b}_{\ell}$ is the empirically estimated bias coefficient, and $b_{\ell}\left(\theta^{*}\right)$ is the theoretically predicted bias coefficient; $\theta^{*}$ denotes the parameter value that minimizes the sum of squared residuals. The estimated process for inflation is $x_{t}=0.83 x_{t-1}+\varepsilon_{t}$. The precise descriptions of the models are given in Section 3 and Appendix A.

the standard errors by both individual forecaster and quarter, as in Thompson (2011) and Colin Cameron, Gelbach, and Miller (2011).

The pattern of the estimated bias coefficients is very similar to that obtained using the consensus forecasts. However, the bias coefficient at lag 3 is no longer statistically significant at the $5 \%$ level $(p=0.096)$. Overall, the point estimates from the individuallevel data are somewhat larger in absolute value. However, they fall well within the confidence intervals obtained using consensus forecasts. Of course, the result is not unexpected given the theoretical aggregation result in Section 4.2.

\subsection{Calibration Exercise}

The estimated bias coefficients can be used to guide theory. To illustrate this point, we perform a simple calibration exercise. For a number of models, we choose their parameters to fit the estimated bias coefficients as closely as possible. Specifically, for each model of expectations, we choose its parameters $\theta$ to minimize the sum of squared residuals

$$
\mathrm{SSR}=\sum_{\ell=1}^{12}\left[\hat{b}_{\ell}-b_{\ell}(\theta)\right]^{2},
$$

where $\hat{b}_{\ell}$ is the empirically estimated bias coefficient, and $b_{\ell}(\theta)$ is the theoretically predicted bias coefficient. To get the theoretical predictions, we assume that the true inflation process is an $\operatorname{AR}(1)$. Estimating the persistence parameter using least squares yields an estimate of $\hat{\rho}=0.83$ (see Table 1 ).

The results from this exercise are shown in Table 2. Since the models all have a single parameter (except for rational expectations which have no free parameters), we 


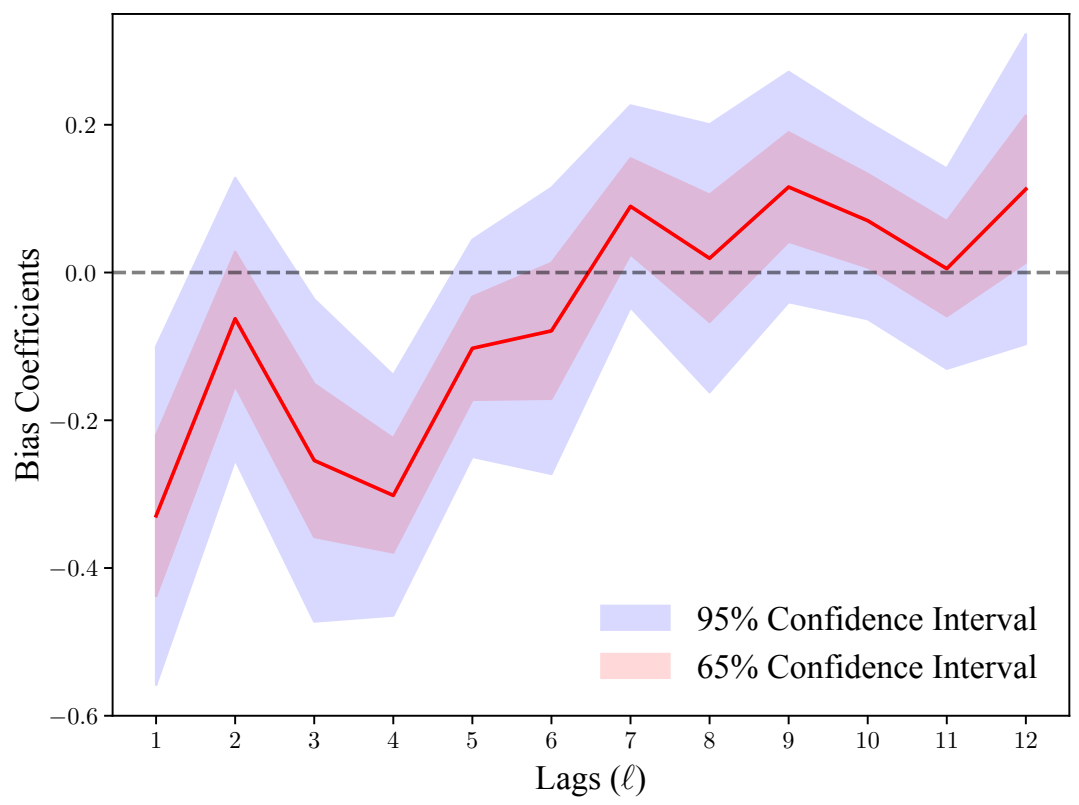

(a) Inflation: Consensus-Level Estimates

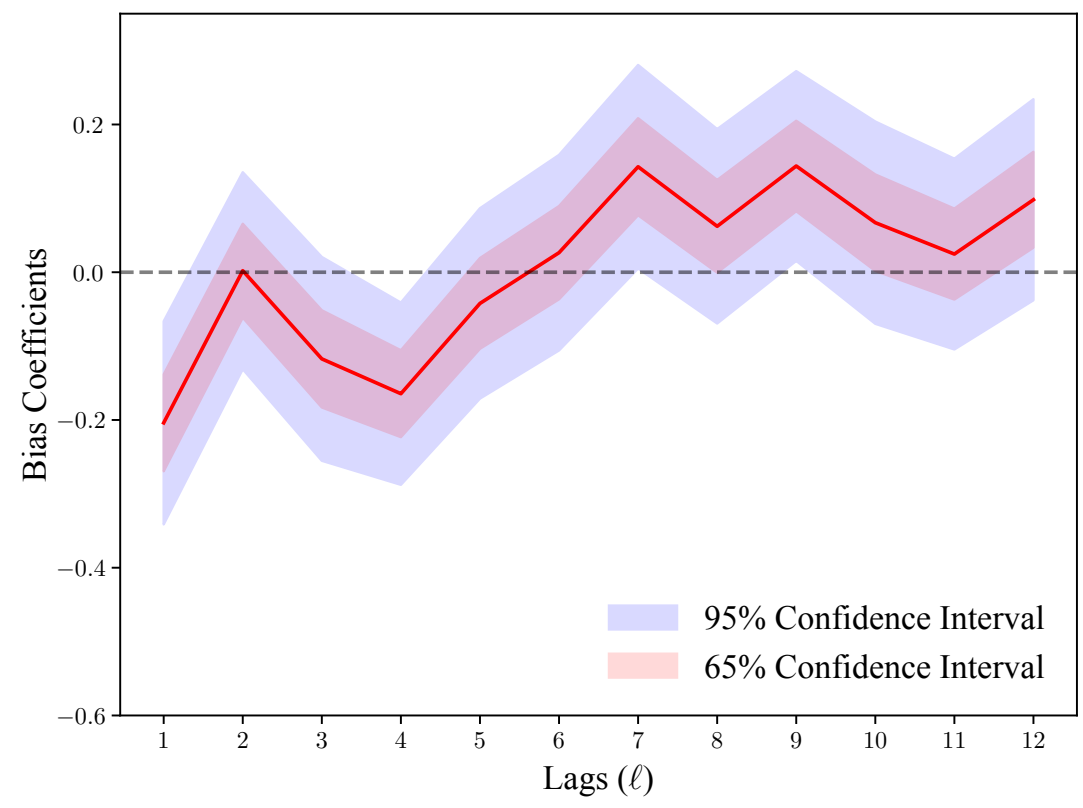

(b) Inflation: Individual-Level Estimates

Figure 5: Bias coefficients for one-quarter ahead inflation forecasts. Top panel: estimates using consensus (median) forecasts; Newey-West standard errors with four lags are used to calculate the confidence intervals. Bottom panel: estimates using individual-level data (controlling for forecaster fixed effects); standard errors are clustered by both forecaster and quarter. Both sets of estimates are obtained by first using local projections (with $K=4$ ) to estimate the impulse response function of the forecast errors:

$$
x_{t+s}-\mathbb{F}_{t+s-1}\left[x_{t+s}\right]=\alpha+\beta_{1}^{(s)}\left\{x_{t-1}-\mathbb{F}_{t-2}\left[x_{t-1}\right]\right\}+\cdots+\beta_{4}^{(s)}\left\{x_{t-4}-\mathbb{F}_{t-3}\left[x_{t-4}\right]\right\}+u_{t},
$$

where $s=0,1, \ldots, 11$. The bias coefficients are then estimated by $\hat{b}_{\ell}=-\hat{\beta}_{1}^{(\ell-1)}$. 
do not adjust for model complexity. The model that best fits the data is a simple model in which forecasters think that the true persistence of inflation is smaller than it actually is (see Section A.3). The perceived level of persistence that provides the best fit is 0.61 . This number is roughly $25 \%$ lower than the estimated persistence of inflation. As discussed by Gabaix (2017a, pp. 14-15), limited attention can naturally lead to a misperception of persistence.

The sticky information model also does well, with only a slightly worse fit than the misperception model. The estimated information stickiness parameter for this model is $\hat{\lambda}=0.51$. The estimate is very close to that reported by Coibion and Gorodnichenko (2015) who find $\hat{\lambda}=0.54$ (s.e. $=0.10) .{ }^{35}$ While Coibion and Gorodnichenko (2015) also use the SPF data, they consider one-year ahead forecasts, and their methodology estimates the level of stickiness by regressing forecast errors on past forecast revisions. The fact that we get a very similar number using a completely different methodology is reassuring. In the context of the sticky information model, our estimate implies that forecasters in the SPF update their information sets roughly twice a year on average.

The model with adjustment costs performs better than simple rational expectations. However, in the current setting it is not exactly clear what the adjustment cost may represent, given that the identities of the participants are not publicly known. Diagnostic expectations in this example do exactly as well as rational expectations, since they cannot generate underreaction. Finally, mechanical adaptive and extrapolative expectations perform worse than rational expectations. The finding is consistent with previous research that has documented that participants in the SPF are quite accurate. ${ }^{36}$ It is therefore not surprising that their behavior is not very well described by mechanical models of expectations. The fact that extrapolative expectations perform especially poorly is interesting in light of the fact that extrapolative models explain inflation expectations well in laboratory experiments (e.g., Pfajfar and Žakelj, 2014).

\section{Conclusions}

We have developed a new framework for measuring biases in expectation formation. The framework allows for a fairly unified treatment of existing models, and it provides a simple way to measure biases empirically. The estimated bias coefficients can be used to distinguish between different models of expectations. Since our empirical method

\footnotetext{
${ }^{35}$ We use the Delta Method to calculate the standard error for $\hat{\lambda}$ from the estimates provided by Coibion and Gorodnichenko, i.e., s.e. $(\hat{\lambda})=$ s.e. $(\hat{\beta}) /(1+\hat{\beta})^{2}$.

${ }^{36} \mathrm{See}$, for example, Croushore (2010). Croushore documents that the median forecast in the SPF performs better than simple time series models of inflation (Table 5), and that it is difficult to adjust forecasts for biases observed in the past to obtain higher forecasting accuracy (Table 4).
} 
is based on local projections, it is easy to accommodate various empirical scenarios, including panel data and clustered error terms. The flexibility of local projections can also be brought to bear on questions that may be difficult to address otherwise, such as whether expectations exhibit non-linearities or state dependence.

The proposed method imposes fewer assumptions than existing approaches. The additional generality can come at the cost of lower statistical precision. When considering using our method, researchers should weigh the costs of imposing additional assumptions - which may be violated in practice - against the benefits of increased statistical power. Additional structure is likely to be especially valuable when the dataset has a small time series dimension.

The notion of bias used in this paper is a statistical one. There may be multiple reasons for why expectations are biased according to this definition, including psychological (e.g., belief persistence) as well as non-psychological ones (e.g., informational frictions). A related challenge is that different models of expectations can yield similar predictions for bias coefficients. Predictions on additional moments of the data can help to distinguish between competing explanations in these situations. ${ }^{37}$

Given the methodological nature of the present work, we have illustrated the method with a single yet prominent application: inflation expectations. Many new datasets on subjective expectations have become available in recent years, thanks to numerous data collection efforts. While much remains to be done, new data sources will no doubt shed light on how expectations are formed. Our method may prove useful in this endeavor.

\footnotetext{
${ }^{37}$ For instance, in Section 3, the models of sticky information and noisy information are shown to imply identical values for the bias coefficients when the underlying process is an AR(1). However, the two models make different predictions on how disagreement among forecasters changes in response to shocks (Coibion and Gorodnichenko, 2012).
} 


\section{References}

Abarbanell, J. S., Bernard, V. L., 1992. Tests of analysts' overreaction/underreaction to earnings information as an explanation for anomalous stock price behavior. The Journal of Finance 47 (3), 1181-1207.

Andreassen, P. B., 1987. On the social psychology of the stock market: Aggregate attributional effects and the regressiveness of prediction. Journal of Personality and Social Psychology 53 (3), 490-496.

Assenza, T., Bao, T., Hommes, C., Massaro, D., 2014. Experiments on expectations in macroeconomics and finance. In: Duffy, J. (Ed.), Experiments in Macroeconomics (Research in Experimental Economics). Vol. 17. Emerald Group Publishing Limited, Bingley, pp. 11-70.

Bao, T., Hommes, C., Makarewicz, T., 2017. Bubble formation and (in)efficient markets in learning-to-forecast and optimise experiments. The Economic Journal 127, 581609.

Barberis, N., Greenwood, R., Jin, L., Shleifer, A., 2015. X-CAPM: An extrapolative capital asset pricing model. Journal of Financial Economics 115 (1), 1-24.

Barberis, N., Shleifer, A., Vishny, R., 1998. A model of investor sentiment. Journal of Financial Economics 49 (3), 307-343.

Blackwell, D., Dubins, L., 1962. Merging of opinions with increasing information. The Annals of Mathematical Statistics 33 (3), 882-886.

Bordalo, P., Gennaioli, N., Shleifer, A., 2018. Diagnostic expectations and credit cycles. The Journal of Finance 73 (1), 199-227.

Bouchaud, J.-P., Krüger, P., Landier, A., Thesmar, D., 2018. Sticky expectations and the profitability anomaly. The Journal of Finance, forthcoming.

Brock, W. A., Hommes, C., 1997. A rational route to randomness. Econometrica 65 (5), 1059-1095.

Cagan, P., 1956. The monetary dynamics of hyperinflation. In: Friedman, M. (Ed.), Studies in the Quantity Theory of Money. University of Chicago Press, Chicago, pp. 25-117.

Capistrán, C., Timmerman, A., 2009. Disagreement and biases in inflation expectations. Journal of Money, Credit and Banking 41 (2), 365-396. 
Carroll, C. D., 2003. Macroeconomic expectations of households and professional forecasters. The Quarterly Journal of Economics 118 (1), 269-298.

Chapman, L. J., Chapman, J., 1982. Test results are what you think they are. In: Kahneman, D., Slovic, P., Tversky, A. (Eds.), Judgment under Uncertainty. Cambridge University Press, Cambridge, Ch. 17.

Cochrane, J., 2005. Asset Pricing: Revised Edition. Princeton University Press, Princeton.

Coibion, O., Gorodnichenko, Y., 2012. What can survey forecasts tell us about information rigidities? Journal of Political Economy 120 (1), 116-159.

Coibion, O., Gorodnichenko, Y., 2015. Information rigidity and the expectations formation process: A simple framework and new facts. The American Economic Review 105 (8), 2644-2678.

Coibion, O., Gorodnichenko, Y., Kamdar, R., 2018. The formation of expectations, inflation and the Phillips Curve. Journal of Economic Literature, forthcoming.

Colin Cameron, A., Gelbach, J. B., Miller, D. L., 2011. Robust inference with multiway clustering. Journal of Business and Economic Statistics 29 (2), 238-249.

Croushore, D., 2010. An evaluation of inflation forecasts from surveys using real-time data. The B.E. Journal of Macroeconomics 10 (1).

Croushore, D. D., 1993. Introducing: The Survey of Professional Forecasters. Business Review - Federal Reserve Bank of Philadelphia 3 (December), 3-15.

Cutler, D. M., Poterba, J. M., Summers, L. H., 1990a. Speculative dynamics. Review of Economic Studies 58 (3), 529-546.

Cutler, D. M., Poterba, J. M., Summers, L. H., 1990b. Speculative dynamics and the role of feedback traders. The American Economic Review: Papers \& Proceedings 80 (2), 63-68.

Daniel, K., Hirshleifer, D., Subrahmanyam, A., 1998. Investor psychology and security market under- and overreactions. The Journal of Finance 53 (6), 1839-1885.

De Bondt, W. F. M., Thaler, R. H., 1990. Do security analysts overreact? The American Economic Review: Papers \& Proceedings 80 (2), 52-57. 
DeLong, B. J., Shleifer, A., Summers, L. H., Waldmann, R. J., 1990. Positive feedback investment strategies and destabilizing rational speculation. The Journal of Finance 45 (2), 379-395.

Diebold, F. X., Lopez, J. A., 1996. Forecast evaluation and combination. In: Maddala, G., Rao, C. (Eds.), Handbook of Statistics (Statistical Methods in Finance). Vol. 14. Elsevier, Amsterdam, Ch. 8, pp. 241-268.

Dominitz, J., Manski, C. F., 2011. Measuring and interpreting expectations of equity returns. Journal of Applied Econometrics 26, 352-370.

Durbin, J., Koopman, S. J., 2012. Time Series Analysis by State Space Methods. Oxford University Press, Oxford.

Edwards, W., 1968. Conservatism in human information processing. In: Kleinmuntz, B. (Ed.), Formal Representation of Human Judgement. John Wiley and Sons, New York.

Evans, G. W., Honkapohja, S., 2001. Learning and Expectations in Macroeconomics. Princeton University Press, Princeton.

Federal Reserve Bank of Philadelphia, 2017. Survey of Professional Forecasters: Documentation. Link to website.

Fisher, F., 1962. A Priori Information and Time Series Analysis: Essays in Economic Theory and Measurement. Amsterdam: North-Holland.

Fuster, A., Laibson, D., Mendel, B., 2010. Natural expectations and macroeconomic fluctuations. Journal of Economic Perspectives 24 (4), 67-84.

Gabaix, X., 2017a. Behavioral inattention. Working Paper, Harvard University.

Gabaix, X., 2017b. Behavioral macroeconomics via sparse dynamic programming. Working Paper, Harvard University.

Gabaix, X., 2018. A behavioral New Keynesian model. Working Paper, Harvard University.

Gennaioli, N., Shleifer, A., 2010. What comes to mind. The Quarterly Journal of Economics 125 (4), 1399-1433.

Gilovich, T., Vallone, R., Tversky, A., 1985. The hot hand in basketball: On the misperception of random sequences. Cognitive Psychology 17, 295-314. 
Goodwin, R. M., 1947. Dynamical coupling with especial reference to markets having production lags. Econometrica 15 (3), 181-204.

Graham, J. R., Harvey, C. R., 2001. Expectations of equity risk premia, volatility and asymmetry from a corporate finance perspective. NBER Working Paper No. 8678.

Greenwood, R., Shleifer, A., 2014. Expectations of returns and expected returns. Review of Financial Studies 27 (3), 714-746.

Griffin, D., Tversky, A., 1992. The weighing of evidence and the determinants of confidence. Cognitive Psychology 24, 411-435.

Hamilton, J. D., 1994. Time Series Analysis. Princeton University Press, Princeton.

Hannan, E. J., 1970. Multiple Time Series. John Wiley and Sons.

Heemeijer, P., Hommes, C., Sonnemans, J., Tuinstra, J., 2009. Price stability and volatility in markets with positive and negative expectations feedback: An experimental investigation. Journal of Economic Dynamics \& Control 33, 1052-1072.

Hey, J. D., 1994. Expectations formation: Rational or adaptive or ...? Journal of Economic Behavior and Organization 25 (3), 329-349.

Jordá, Ò., 2005. Estimation and inference of impulse responses by local projections. The American Economic Review 95 (1), 161-182.

Kahneman, D., Tversky, A., 1973. On the psychology of prediction. Psychological Review 80 (4), 237-251.

Keane, M. P., Runkle, D. E., 1990. Testing the rationality of price forecasts: New evidence from panel data. The American Economic Review 80 (4), 714-735.

Koop, G., Pesaran, M., Potter, S. M., 1996. Impulse response analysis in nonlinear multivariate models. Journal of Econometrics 74 (1), 119-147.

Kydland, F. E., Prescott, E. C., 1982. Time to build and aggregate fluctuations: Some new evidence. Econometrica 50 (6), 1345-1370.

Langer, E. J., 1975. The illusion of control. Journal of Personality and Social Psychology $32,311-328$.

Ma, Y., Landier, A., Thesmar, D., 2017. New experimental evidence on expectations formation. Working Paper. 
Mankiw, G. N., Reis, R., 2002. Sticky information versus sticky prices: A proposal to replace the New Keynesian Phillips Curve. The Quarterly Journal of Economics 117 (4), 1295-1328.

Mankiw, N. G., Reis, R. A., Wolfers, J., 2003. Disagreement about inflation expectations. NBER Macroeconomics Annual 18, 209-270.

Manski, C. F., 2004. Measuring expectations. Econometrica 72 (5), 1329-1376.

Manski, C. F., 2018. Survey measurement of probabilistic macroeconomic expectations: Progress and promise. NBER Macroeconomics Annual 32, forthcoming.

Marimon, R., 1997. Learning from learning in economics. In: Kreps, D. M., Wallis, K. F. (Eds.), Advances in Economics and Econometrics: Theory and Applications. Vol. 1. Cambridge University Press, Cambridge, Ch. 9, pp. 278-315.

Miller, J. B., Sanjurjo, A., 2018. Surprised by the hot hand fallacy? A truth in the law of small numbers. Econometrica, forthcoming.

Mincer, J. A., Zarnowitz, V., 1969. The evaluation of economic forecasts. In: Mincer, J. A. (Ed.), Economic Forecasts and Expectations: Analysis of Forecasting Behavior and Performance. National Bureau of Economic Research, Ch. 1, pp. 3-46.

Muth, J. F., 1961. Rational expectations and the theory of price movements. Econometrica 29 (3), 315-335.

Nerlove, M., 1958. Adaptive expectations and cobweb phenomena. The Quarterly Journal of Economics 72 (2), 227-240.

Nickerson, R. S., 1998. Confirmation bias: A uniquitous phenomenon in many guises. Review of General Psychology 2 (2), 175-220.

Nisbett, R., Ross, L., 1980. Human Inference: Strategies and Shortcomings of Social Judgement. Prentice Hall, Englewood Cliffs.

Odean, T., 1998. Volume, volatility, price, and profit when all traders are above average. The Journal of Finance 53 (6), 1887-1934.

Patton, A. J., Timmermann, A., 2007. Properties of optimal forecasts under asymmetric loss and nonlinearity. Journal of Econometrics 140 (2), 884-918.

Pesaran, M. H., Weale, M., 2006. Survey expectations. In: Elliott, G., Granger, C. W. J., Timmermann, A. (Eds.), Handbook of Economic Forecasting. Vol. 1. Elsevier, Amsterdam, Ch. 14, pp. 715-776. 
Pfajfar, D., Žakelj, B., 2014. Experimental evidence on inflation expectation formation. Journal of Economic Dynamics \& Control 44, 147-168.

Ramey, V. A., 2016. Macroeconomic shocks and their propagation. In: Taylor, J. B., Uhlig, H. (Eds.), Handbook of Macroeconomics. Vol. 2. Elsevier, Amsterdam, Ch. 2 , pp. $71-162$.

Reis, R., 2006. Inattentive producers. Review of Economic Studies 73 (3), 793-821.

Schmalensee, R., 1976. An experimental study of expectation formation. Econometrica 44 (1), 17-41.

Sims, C. A., 2003. Implications of rational inattention. Journal of Monetary Economics $50(3), 665-690$.

Staudenmayer, J., Buonaccorsi, J. P., 2005. Measurement error in linear autoregressive models. Journal of the American Statistical Association 100, 841-852.

Thompson, S. B., 2011. Simple formulas for standard errors that cluster by both firm and time. Journal of Financial Economics 99 (1), 1-10.

Vissing-Jorgensen, A., 2004. Perspectives on behavioral finance: Does 'irrationality' disappear with wealth? Evidence from expectations and actions. NBER Macroeconomics Annual 18, 139-208.

Woodford, M., 2003. Imperfect common knowledge and the effects of monetary policy. In: Aghion, P., Frydman, R., Stiglitz, J., Woodford, M. (Eds.), Knowledge, Information, and Expectations in Modern Macroeconomics: In Honor of Edmund S. Phelps. Princeton University Press, Princeton, pp. 25-58. 


\section{Appendix A Mapping Existing Models}

\section{A.1 Adaptive Expectations}

Suppose that agent has adaptive expectations as in Cagan (1956) and Nerlove (1958):

$$
\mathbb{F}_{t}\left[x_{t+1}\right]=\mathbb{F}_{t-1}\left[x_{t}\right]+\lambda\left\{x_{t}-\mathbb{F}_{t-1}\left[x_{t}\right]\right\}
$$

Iterating, we have that

$$
\begin{aligned}
\mathbb{F}_{t}\left[x_{t+1}\right]=\lambda \sum_{\ell=0}^{+\infty}(1-\lambda)^{\ell} x_{t-\ell} & =\lambda \sum_{\ell=0}^{+\infty} \sum_{j=0}^{+\infty}(1-\lambda)^{\ell} \rho^{j} \varepsilon_{t-\ell-j} \\
& =\lambda \sum_{\ell=0}^{+\infty}\left(\frac{(1-\lambda)^{\ell+1}-\rho^{\ell+1}}{1-\lambda-\rho}\right) \varepsilon_{t-\ell} .
\end{aligned}
$$

Hence, we obtain

$$
\begin{aligned}
& a_{\ell}=\lambda\left[\frac{(1-\lambda)^{\ell}-\rho^{\ell}}{1-\lambda-\rho}\right] \\
& b_{\ell}=\operatorname{sgn}\left(\rho^{\ell}\right)\left[\frac{\lambda(1-\lambda)^{\ell}-(1-\rho) \rho^{\ell}}{1-\lambda-\rho}\right]
\end{aligned}
$$

\section{A.2 Noisy Information}

We now analyze a model in which agents are rational and understand the structure of the model but do not observe the underlying state perfectly. Models of this type include the rational inattention model of Sims (2003) and the imperfect information model studied by Woodford (2003). The section follows Coibion and Gorodnichenko (2015, pp. 2649 2650) closely.

Suppose the true process for $x_{t}$ is an AR(1) but each agent $i$ only observes a noisy signal $y_{i t}$ of $x_{t}$ :

$$
y_{i t}=x_{t}+\omega_{i t}
$$

Here, $\omega_{i t}$ is a normally distributed mean-zero noise term which is i.i.d. across time and agents. The Kalman filter equations then imply (see Eq. 8 in Coibion and Gorodnichenko (2015)) that

$$
\mathbb{F}_{i t}\left[x_{t+1}\right]=\rho\left\{G y_{i t}+(1-G) \mathbb{F}_{i, t-1}\left[x_{t}\right]\right\}
$$

where $G \in[0,1]$ is the Kalman gain. 
Assuming that there is a continuum of agents and making the usual Law of Large Numbers assumption, we can integrate over agents to find

$$
\mathbb{F}_{t}\left[x_{t+1}\right]=G \rho x_{t}+(1-G) \rho \mathbb{F}_{t-1}\left[x_{t}\right]
$$

with $\mathbb{F}_{t}\left[x_{t+1}\right]$ denoting the average forecast across agents.

Now write $\mathbb{F}_{t}\left[x_{t+1}\right]=\sum_{\ell=0}^{+\infty} a_{\ell+1} \varepsilon_{t-\ell}$ for some unknown coefficients to be determined. From Eq. (13), we have that

$$
a_{1} \varepsilon_{t}+\sum_{\ell=1}^{+\infty}\left[a_{\ell+1}-(1-G) \rho a_{\ell}\right] \varepsilon_{t-\ell}=G \rho \varepsilon_{t}+G \sum_{\ell=1}^{+\infty} \rho^{\ell+1} \varepsilon_{t-\ell}
$$

Matching coefficients and solving the resulting difference equation, we find that

$$
\begin{aligned}
& a_{\ell}=\rho^{\ell}-[(1-G) \rho]^{\ell} \\
& b_{\ell}=-\operatorname{sgn}\left(\rho^{\ell}\right)[(1-G) \rho]^{\ell}
\end{aligned}
$$

Hence, as long as the signal is not perfectly revealing of the state $(G \neq 1)$, the noisy information model predicts underreaction. In fact, the model predicts identical bias coefficients as the sticky information model when $\lambda=1-G$.

\section{A.3 Misperceived Law of Motion}

Suppose that the agent misperceives the true persistence of the process and makes forecasts as

$$
\mathbb{F}_{t}\left[x_{t+1}\right]=\hat{\rho} x_{t}, \hat{\rho} \in(-1,1)
$$

with $\hat{\rho}$ potentially different from $\rho$. Examples of models with misperceived laws of motion abound in the literature, with two prominent cases given by Barberis, Shleifer, and Vishny (1998) and Fuster, Laibson, and Mendel (2010); see also Gabaix (2017a, pp. 14-15). In the present case,

$$
\mathbb{F}_{t}\left[x_{t+1}\right]=\hat{\rho} \sum_{\ell=0}^{+\infty} \rho^{\ell} \varepsilon_{t-\ell}
$$

and therefore

$$
\begin{aligned}
& a_{\ell}=\hat{\rho} \rho^{\ell-1} \\
& b_{\ell}=\operatorname{sgn}\left(\rho^{\ell}\right)\left[\hat{\rho} \rho^{\ell-1}-\rho^{\ell}\right]
\end{aligned}
$$


When $\rho \neq 0$, we can write

$$
b_{\ell}=\operatorname{sgn}\left(\rho^{\ell}\right) \rho^{\ell}\left(\frac{\hat{\rho}-\rho}{\rho}\right)
$$

If $\rho>0$, the agent overreacts to news whenever $\hat{\rho}>\rho$ and underreacts otherwise.

\section{A.4 Extrapolative Expectations}

We now consider pure extrapolative expectations

$$
\mathbb{F}_{t}\left[x_{t+1}\right]=x_{t}+\gamma\left(x_{t}-x_{t-1}\right),
$$

as in Goodwin (1947, p. 191). The parameter $\gamma$ could be either positive or negative, with a positive $\gamma$ representing extrapolation or trend following, while a negative $\gamma$ could capture contrarian expectations.

Substituting in the expression for $x_{t}$, we calculate that

$$
\mathbb{F}_{t}\left[x_{t+1}\right]=(1+\gamma) \varepsilon_{t}+\sum_{\ell=1}^{+\infty}\left\{(1+\gamma) \rho^{\ell}-\gamma \rho^{\ell-1}\right\} \varepsilon_{t-\ell}
$$

and so we find that

$$
a_{\ell}=\left\{\begin{array}{ll}
1+\gamma & \text { if } \ell=1 \\
(1+\gamma) \rho^{\ell-1}-\gamma \rho^{\ell-2} & \text { if } \ell \geq 2
\end{array},\right.
$$

and

$$
b_{\ell}= \begin{cases}\operatorname{sgn}(\rho)(1+\gamma-\rho) & \text { if } \ell=1 \\ \operatorname{sgn}\left(\rho^{\ell}\right)\left\{(1+\gamma) \rho^{\ell-1}-\gamma \rho^{\ell-2}-\rho^{\ell}\right\} & \text { if } \ell \geq 2\end{cases}
$$

Suppose expectations are of the trend-following type $(\gamma>0)$. Then, if $x_{t}$ is positively autocorrelated, the agent always overreacts to current news $\left(b_{1}>0\right)$. However, extrapolative expectations may well lead to underreaction to past news.

\section{A.5 Learning}

Many models of expectations study agents that are learning the true data-generating process over time. These models naturally lead to time-varying expectations. As a result, models with learning are generally not nested by our time-invariant formulation in 
Eq. (2). We could add a time $t$ subscript to our specification without any theoretical difficulty. The problem is a practical one. Without assuming some constancy of parameters or imposing some other structure on expectations, measuring bias coefficients empirically does not seem feasible.

Models of learning often have the property that agents eventually learn the true model. According to the Blackwell-Dubins Theorem (Blackwell and Dubins, 1962), that is the case if agents are Bayesian, and - very loosely speaking-place a positive prior probability on the true model. ${ }^{38}$ Another example is if the agents do not know the true data-generating process but estimate correctly specified regressions, as in many models of adaptive learning (Evans and Honkapohja, 2001). In these circumstances, a natural empirical approach is to split the sample into early and late periods and estimate the bias coefficients separately in the two subsamples. The prediction of learning is that the estimated bias coefficients should be closer to zero in the later subsample.

Finally, in some models, the agents know the true model but are not able to observe the true state. Such models involve learning about the true state (although not the parameters of the model). For example, the variable being forecast may consist of a transitory and a permanent component, but the agent may not be able to distinguish between the two. An example is given by the seminal paper by Kydland and Prescott (1982) who assume that the technology shock consists of a permanent and transitory component (see their equation 3.7). If the model can be written as a linear state space model with normally distributed disturbances, then a key result in the theory of Kalman filtering states that the forecast errors are independent. ${ }^{39}$ In these cases, the predicted bias coefficients are zero.

\section{A.6 Overconfidence}

Finally, we discuss a stylized model of overconfidence along the lines of Daniel, Hirshleifer, and Subrahmanyam (1998) and Odean (1998), among others. Suppose that expectations follow

$$
\mathbb{F}_{t}\left[x_{t+1}\right]=s_{t},
$$

where $s_{t}$ is white noise (and independent of $\varepsilon_{t}$ ). We think of this specification as capturing an extreme form of overconfidence. In this interpretation, $s_{t}$ is a private signal that is uncorrelated with the actual news.

According to the definition in Section 4.4, expectations simultaneously overreact to the private signal $\left(s_{t}\right)$ and underreact to the actual news $\left(\varepsilon_{t}\right)$. Overall, expectations

\footnotetext{
${ }^{38}$ Marimon (1997, pp. 285-286) discusses this result and its implications in economics.

${ }^{39}$ See, for example, Durbin and Koopman (2012, p. 69).
} 
exhibit underreaction. To see this, note that we can write $(1-\rho L) e_{t}=\varepsilon_{t}-s_{t-1}+\rho s_{t-2}$. The right-hand side is a sum of an MA(1) process and white noise, and therefore also an MA(1) process (see, e.g., Hamilton, 1994, pp. 102-105). Write the right-hand side as $\xi_{t}+\theta \xi_{t-1}$ for some $\theta$ and a white noise series $\xi_{t}$. Then, the Wold Representation of $e_{t}$ is given by ${ }^{40}$

$$
e_{t}=\xi_{t}+(\rho+\theta) \sum_{\ell=1}^{+\infty} \rho^{\ell-1} \xi_{t-\ell} .
$$

One can show that $\rho+\theta>0$, and therefore expectations exhibit overall underreaction. As these calculations make clear, the present overconfidence model is isomorphic to the model with a misperceived law of motion (Section A. 3 with $\hat{\rho}=0$ ) and measurement error. Empirically, it may be challenging to disentangle these two possibilities.

\footnotetext{
${ }^{40}$ The calculation is exactly the same as that used to calculate the bias coefficients in the presence of measurement error in Appendix B.1, and the expression for $\theta$ is given in Eq. (16) with $(\lambda \rho)$ replaced with $\rho$.
} 


\section{Appendix B Measurement Error}

\section{B.1 Formulas for Sticky and Diagnostic Expectations}

This appendix provides explicit formulas for the attenuation bias caused by measurement error for two models of expectations (sticky information and diagnostic expectations). As in Section 3, $x_{t}$ follows a stationary AR(1) process.

First, suppose that the true expectations are generated by the sticky information model of Mankiw and Reis (2002), implying that the true forecast errors follow

$$
e_{t}=\lambda \rho e_{t-1}+\varepsilon_{t}
$$

However, instead of observing the true forecast $\mathbb{F}_{t}\left[x_{t+1}\right]$, we can only observe

$$
\mathbb{F}_{t}^{*}\left[x_{t+1}\right]=\mathbb{F}_{t}\left[x_{t+1}\right]+v_{t}
$$

where $v_{t}$ is white noise measurement error with variance $\sigma_{v}^{2}$ (and independent of $\varepsilon_{t}$ ). The observed forecast error is then equal to $e_{t}^{*}=e_{t}-v_{t-1}$. Now write

$$
e_{t}^{*}-\lambda \rho e_{t-1}^{*}=\varepsilon_{t}-v_{t-1}+\lambda \rho v_{t-2} .
$$

The right-hand side of Eq. (15) is the sum of an MA(1) process and white noise, and therefore also an MA(1) process (see, e.g., Hamilton, 1994, pp. 102-105). Denote the resulting process as $\xi_{t}+\theta \xi_{t-1}$ for some parameters $\theta$ and $\sigma_{\xi}^{2}$ to be determined. For the representation to be valid, the autocovariances must match, namely

$$
\begin{aligned}
\sigma_{\varepsilon}^{2}+\left[1+(\lambda \rho)^{2}\right] \sigma_{v}^{2} & =\left(1+\theta^{2}\right) \sigma_{\xi}^{2} \\
-\lambda \rho \sigma_{v}^{2} & =\theta \sigma_{\xi}^{2}
\end{aligned}
$$

Substituting out $\sigma_{\xi}$ and rearranging leads to a quadratic equation in $\theta$ :

$$
(-\lambda \rho) \theta^{2}-\theta\left\{\frac{\sigma_{\varepsilon}^{2}}{\sigma_{v}^{2}}+\left[1+(\lambda \rho)^{2}\right]\right\}-\lambda \rho=0 .
$$

The equation has two real solutions. Picking the solution associated with the invertible representation (i.e., with $|\theta|<1$ ) yields

$$
\theta=\frac{\left\{\frac{\sigma_{\varepsilon}^{2}}{\sigma_{v}^{2}}+\left[1+(\lambda \rho)^{2}\right]\right\}-\sqrt{\left\{\frac{\sigma_{\varepsilon}^{2}}{\sigma_{v}^{2}}+\left[1+(\lambda \rho)^{2}\right]\right\}^{2}-4(\lambda \rho)^{2}}}{-2 \lambda \rho}
$$


All in all, the observed forecast errors follow an $\operatorname{ARMA}(1,1)$ process with

$$
e_{t}^{*}(1-\lambda \rho L)=(1+\theta L) \xi_{t}
$$

where $L$ is the lag operator. As a result, the Wold representation of $e_{t}^{*}$ is

$$
e_{t}^{*}=\xi_{t}+(\lambda \rho+\theta) \sum_{\ell=1}^{+\infty}(\lambda \rho)^{\ell-1} \xi_{t-\ell} .
$$

Therefore, measurement error leads to an attenuation bias. The attenuation bias can be substantial if measurement error is large (i.e., signal-to-noise ratio, $\sigma_{\varepsilon} / \sigma_{v}$, is small). For example, suppose that $\lambda=0.50, \rho=0.75, \sigma_{\varepsilon}=0.25$, and $\sigma_{v}=0.15$. Then, the true bias coefficient $b_{1}$ is equal to $(-\lambda \rho)=-0.375$. In contrast, the bias coefficient in the process with measurement error is equal to

$$
-(\lambda \rho+\theta) \approx-(0.375-0.097)=-0.278
$$

The attenuation bias is roughly $26 \%$ in relative terms. This simple calculation highlights the importance of having datasets in which many forecasters forecast the same variable. By averaging across multiple forecasters, $\sigma_{v}$ can be reduced, thereby diminishing the attenuation bias.

Now consider the case of diagnostic expectations. In that case, the true forecast errors follow an $\mathrm{MA}(1)$ process with $e_{t}=\varepsilon_{t}-\theta \rho \varepsilon_{t-1}$, implying that the observed forecast errors are given by $e_{t}^{*}=\varepsilon_{t}-\theta \rho \varepsilon_{t-1}-v_{t-1}$. The right-hand side again follows an MA(1) process but with different parameters. Write $e_{t}^{*}=\xi_{t}+\psi \xi_{t-1}$ for some parameters $\psi$ and $\sigma_{\xi}^{2}$. Similar calculations to those performed earlier show that

$$
\psi=\frac{\left\{\frac{\sigma_{v}^{2}}{\sigma_{\varepsilon}^{2}}+\left[1+(\theta \rho)^{2}\right]\right\}-\sqrt{\left\{\frac{\sigma_{v}^{2}}{\sigma_{\varepsilon}^{2}}+\left[1+(\theta \rho)^{2}\right]\right\}^{2}-4(\theta \rho)^{2}}}{-2 \theta \rho} .
$$

To gauge the size of the attenuation bias, suppose that $\theta=0.50, \rho=0.75, \sigma_{\varepsilon}=0.25$, and $\sigma_{v}=0.15$. With these parameters, the true bias coefficient is equal to $b_{1}=\theta \rho=$ 0.375 . However, the bias coefficient from the process with measurement error (i.e., $-\psi$ ) is equal to approximately 0.268 . In relative terms, the attenuation bias is roughly $29 \%$. 


\section{B.2 Calibrating the Level of Measurement Error}

We now study how large measurement error is likely to be in the context of inflation expectations (at the consensus level). As a side benefit, the section characterizes the effects of measurement error on the procedure proposed by Coibion and Gorodnichenko (2015).

Coibion and Gorodnichenko (2012) use an empirical strategy that directly estimates the underlying $\varepsilon_{t}$ shocks. They find (p. 154, an average across all their specifications) that $\hat{\lambda}=0.82$. In subsequent work, Coibion and Gorodnichenko (2015) use a different estimation strategy to measure $\lambda$ and find that $\hat{\lambda}=0.54$ (p. 2653). The second strategy is potentially subject to attenuation bias stemming from measurement error (see their Footnote 7 and Online Appendix A).

Suppose that - as an upper bound calculation - we can attribute all of the difference between the two estimates to measurement error in the second approach. Coibion and Gorodnichenko (2015) obtain $\lambda$ by estimating the following regression by least squares:

$$
\underbrace{x_{t}-\mathbb{F}_{t-1}^{*}\left[x_{t}\right]}_{\equiv e_{t}^{*}}=\alpha+\beta\left\{\mathbb{F}_{t-1}^{*}\left[x_{t}\right]-\mathbb{F}_{t-2}^{*}\left[x_{t}\right]\right\}+u_{t}
$$

The stars indicate that the variables are measured with error:

$$
\begin{aligned}
& \mathbb{F}_{t-1}^{*}\left[x_{t}\right]=\mathbb{F}_{t-1}\left[x_{t}\right]+v_{t-1} \\
& \mathbb{F}_{t-2}^{*}\left[x_{t}\right]=\mathbb{F}_{t-2}\left[x_{t}\right]+v_{t-2}
\end{aligned}
$$

where $v_{t}$ is a white noise measurement error with variance $\sigma_{v}^{2}$. As a result, $e_{t}^{*}=e_{t}-v_{t-1}$ (with $e_{t}$ denoting the one-step ahead forecast error).

For the sticky information model, $k$-step ahead forecasts are given by

$$
\mathbb{F}_{t}\left[x_{t+k}\right]=(1-\lambda) \sum_{\ell=0}^{+\infty} \lambda^{\ell} \mathbb{E}_{t-\ell}\left[x_{t+k}\right]=\rho^{k} \sum_{\ell=0}^{+\infty} \rho^{\ell}\left(1-\lambda^{\ell+1}\right) \varepsilon_{t-\ell},
$$

implying that $\mathbb{F}_{t}\left[x_{t+2}\right]=\rho \mathbb{F}_{t}\left[x_{t+1}\right]$. Forecast revisions for $x_{t+1}$ are hence

$$
\mathbb{F}_{t}\left[x_{t+1}\right]-\mathbb{F}_{t-1}\left[x_{t+1}\right]=\rho(1-\lambda) \sum_{\ell=0}^{+\infty}(\rho \lambda)^{\ell} \varepsilon_{t-\ell} .
$$


Since the forecast errors are equal to $e_{t}=\sum_{\ell=0}^{+\infty}(\rho \lambda)^{\ell} \varepsilon_{t-\ell}$, we can write

$$
e_{t}=\varepsilon_{t}+\frac{\lambda}{1-\lambda}\left[\rho(1-\lambda) \sum_{\ell=0}^{+\infty}(\rho \lambda)^{\ell} \varepsilon_{t-1-\ell}\right]=\varepsilon_{t}+\frac{\lambda}{1-\lambda}\left\{\mathbb{F}_{t-1}\left[x_{t}\right]-\mathbb{F}_{t-2}\left[x_{t}\right]\right. \text {. }
$$

Combining these results we have that

$$
\operatorname{Var}\left\{\mathbb{F}_{t-1}\left[x_{t}\right]-\mathbb{F}_{t-2}\left[x_{t}\right]\right\}=\frac{\rho^{2}(1-\lambda)^{2} \sigma_{\varepsilon}^{2}}{1-\lambda^{2} \rho^{2}}
$$

and therefore

$$
\operatorname{plim} \hat{\beta}=\frac{\lambda(1-\lambda) \rho^{2}-\frac{\sigma_{v}^{2}}{\sigma_{\varepsilon}^{2}}\left(1-\lambda^{2} \rho^{2}\right)}{(1-\lambda)^{2} \rho^{2}+2 \frac{\sigma_{v}^{2}}{\sigma_{\varepsilon}^{2}}\left(1-\lambda^{2} \rho^{2}\right)}
$$

Note that there are two reasons why the empirically estimated $\hat{\beta}$ would understate the true level of stickiness. First, there is the usual attenuation bias, as seen in the denominator. However, measurement error also introduces a mechanical negative correlation between forecast errors and past forecast revisions, as captured by the second term in the numerator. As a result, even a small amount of measurement error can lead to substantial bias. If measurement error is severe, the estimated $\hat{\beta}$ may even be negative. Indeed, if $\sigma_{v} \rightarrow+\infty$, plim $\hat{\beta} \rightarrow-1 / 2$. We emphasize that measurement error can also be given a more structural economic interpretation. For instance, measurement error is isomorphic to some models of overconfidence (Section A.6).

We can now ask at what level of measurement error the probability limit above would coincide with the empirically measured $\hat{\beta}$. Coibion and Gorodnichenko (2015, p. 2653) estimate $\hat{\beta}=1.19$. Take $\rho=0.85, \sigma_{\varepsilon}^{2}=1.005$, and $\lambda=0.82$ (using the estimates in Coibion and Gorodnichenko, 2012). Solving for $\sigma_{v}^{2}$, we find that $\sigma_{v} \approx 0.21$. In other words, for the estimates in Coibion and Gorodnichenko (2012) and Coibion and Gorodnichenko (2015) to be consistent, the magnitude of measurement error needs to be roughly a fifth of the magnitude of the true shocks. 


\section{Appendix C Data Appendix}

We download data for the individual responses from the website of the Federal Reserve Bank of Philadelphia (link). The downloaded file contains forecasts of GDP deflator inflation for the past quarter $(P G D P 1)$, current quarter $(P G D P 2)$, and the next four quarters ( $P G D P 3$ up to $P G D P 6$ ), see Federal Reserve Bank of Philadelphia (2017, pp. 20-22).

To construct consensus inflation forecasts, we first calculate the median forecast of $P G D P 2$ and $P G D P 3$ in each quarter. Then, we calculate annualized quarter-on-quarter inflation forecasts as

$$
100\left[\left(\frac{P G D P 3}{P G D P 2}\right)^{4}-1\right] .
$$

The approach follows the standard practice in the Survey of Professional Forecasters. To calculate individual inflation forecasts, we directly use the equation above.

For realizations, we use the Real-Time Data Set for Macroeconomists which is also provided by the Philadelphia Fed (link). We use the first-release data for "Price Index for GNP/GDP (P)." In 1995Q4, the first-release data for inflation is not available. In this period, we use the second-release data.

To match forecasts and actuals, we align the forecasts to the date for which they were made. For example, the one-quarter ahead forecast made in the 1970Q1 survey is matched with the actual inflation reported for 1970Q2. 


\section{Appendix D Additional Results}

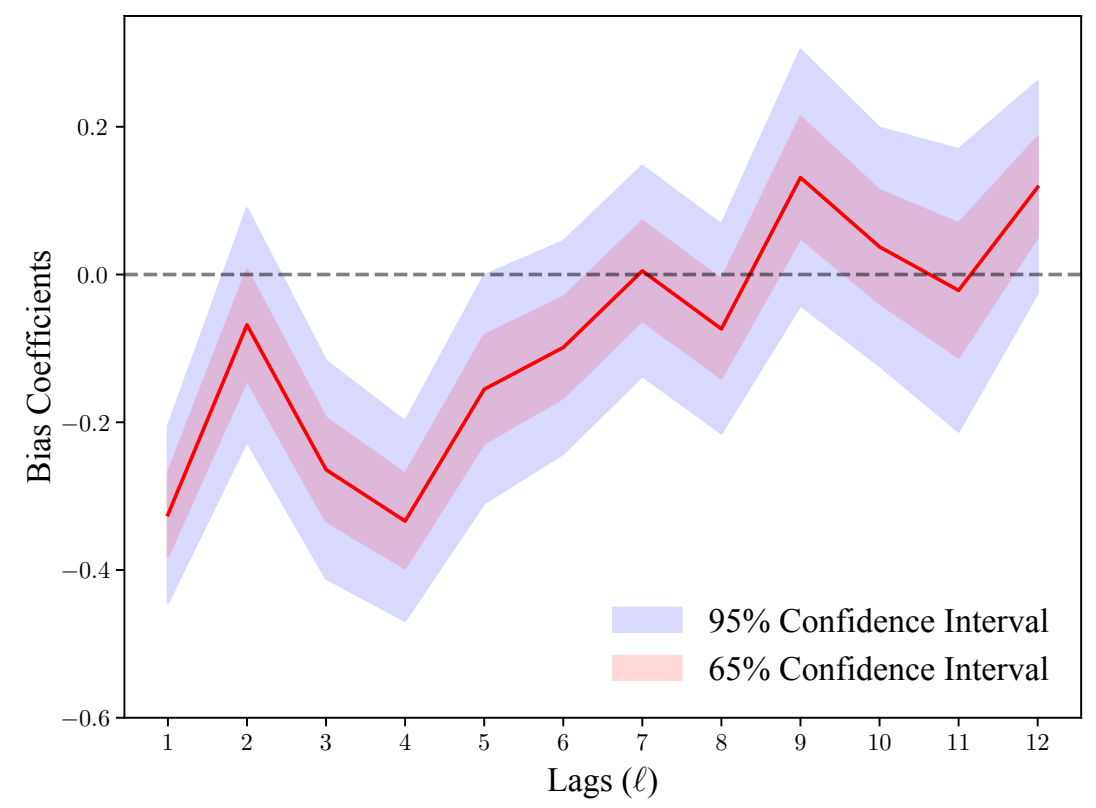

Figure 6: Bias coefficients for one-quarter ahead inflation forecasts: maximum likelihood estimates. The estimation uses consensus (median) forecasts. The impulse response function of the forecast errors is obtained by estimating

$$
x_{t}-\mathbb{F}_{t-1}\left[x_{t}\right]=\varepsilon_{t}+\theta_{1} \varepsilon_{t-1}+\theta_{2} \varepsilon_{t-2}+\cdots+\theta_{12} \varepsilon_{t-12}, \varepsilon_{t} \sim \mathcal{N}\left(0, \sigma_{\varepsilon}^{2}\right)
$$

by maximum likelihood. The bias coefficients are then given by $\hat{b}_{\ell}=-\hat{\theta}_{\ell}$. 


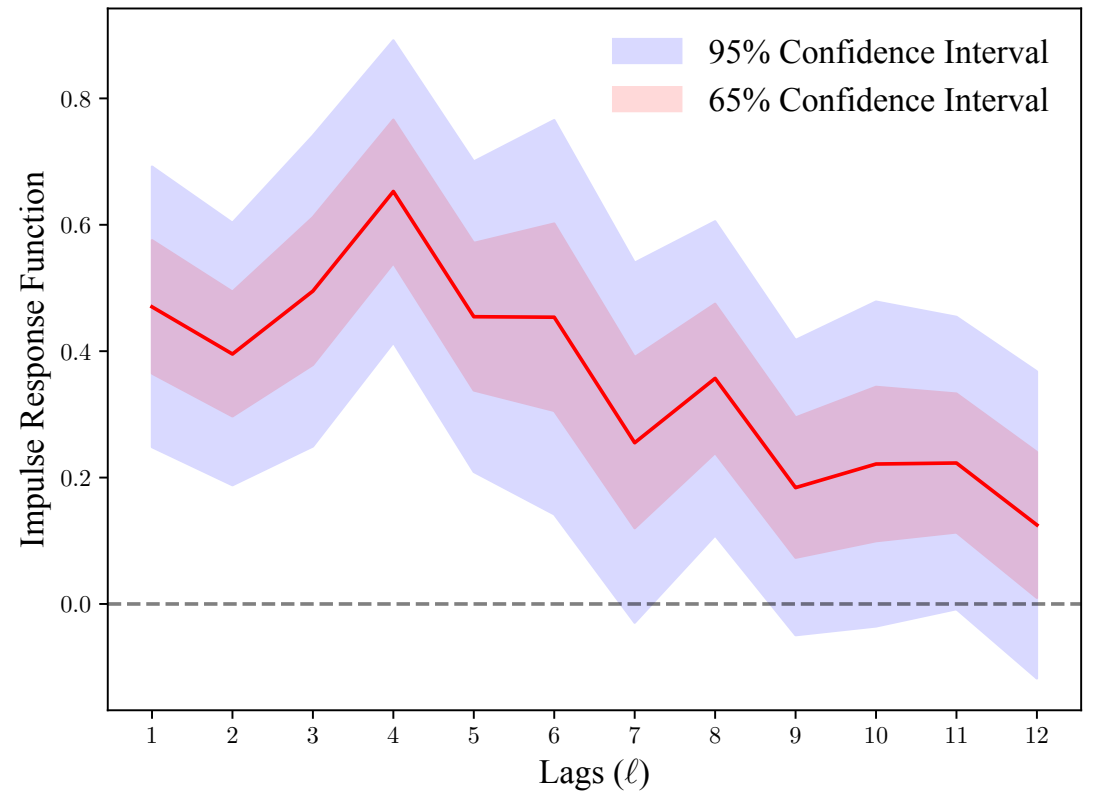

Figure 7: Impulse response function: quarterly GDP deflator inflation. The estimation uses local projections; Newey-West standard errors with four lags are used to calculate the confidence intervals. 